\title{
Stability and monotonicity for some discretizations of the Biot's consolidation model
}

\author{
C. Rodrigo ${ }^{\mathrm{a}, *}$, F.J. Gaspar ${ }^{\mathrm{a}}$, X. Hu ${ }^{\mathrm{b}}$, L.T. Zikatanov ${ }^{\mathrm{c}, \mathrm{d}}$ \\ ${ }^{a}$ Departamento de Matemática Aplicada, Universidad de Zaragoza, Zaragoza, Spain \\ ${ }^{b}$ Department of Mathematics, Tufts University, Medford, Massachusetts 02155, USA \\ ${ }^{c}$ Department of Mathematics, Penn State, University Park, Pennsylvania, 16802, USA \\ ${ }^{d}$ Institute of Mathematics and Informatics, Bulgarian Academy of Sciences, Sofia, \\ Bulgaria
}

\begin{abstract}
We consider finite element discretizations of the Biot's consolidation model in poroelasticity with MINI and stabilized P1-P1 elements. We analyze the convergence of the fully discrete model based on spatial discretization with these types of finite elements and implicit Euler method in time. We also address the issue related to the presence of non-physical oscillations in the pressure approximation for low permeabilities and/or small time steps. We show that even in 1D a Stokes-stable finite element pair fails to provide a monotone discretization for the pressure in such regimes. We then introduce a stabilization term which removes the oscillations. We present numerical results confirming the monotone behavior of the stabilized schemes.
\end{abstract}

Keywords: Stable finite elements, monotone discretizations, poroelasticity.

\section{Introduction}

The theory of poroelasticity models the interaction between the deformation and the fluid flow in a fluid-saturated porous medium. Such coupling was already modelled in the early one-dimensional work of Terzaghi, see [1], whereas the general three-dimensional mathematical model was established by Maurice Biot in several pioneering publications (see [2] and [3]).

\footnotetext{
${ }^{*}$ Corresponding author.

Email addresses: carmenr@unizar.es (C. Rodrigo), fjgaspar@unizar.es (F.J.

Gaspar), xiaozhe.hu@tufts.edu (X. Hu), ltz@math.psu.edu (L.T. Zikatanov)

Preprint submitted to Computer Methods in Applied Mechanics and EngineeringSeptember 22, 2015
}

(C) 2015. This manuscript version is made available under the Elsevier user license http://www.elsevier.com/open-access/userlicense/1.0/ 
We assume here that the porous medium is linearly elastic, homogeneous, isotropic and saturated by an incompressible Newtonian fluid. Under these assumptions, the quasi-static Biot's model can be written as a time-dependent system of partial differential equations in the variables of displacements of the solid, $u$, and pressure of the fluid, $p$,

$$
\begin{aligned}
& -\operatorname{div} \sigma+\nabla p=f, \quad \sigma=2 \mu \varepsilon(u)+\lambda \operatorname{div}(u) I \\
& -\operatorname{div} \dot{u}+\operatorname{div} K \nabla p=g,
\end{aligned}
$$

where $\sigma$ and $\varepsilon$ are the effective stress and strain tensors, $\lambda$ and $\mu$ are the Lamé coefficients, $K$ is the hydraulic conductivity tensor, the right-hand term $f$ is the density of applied body forces and the source term $g$ represents a forced fluid extraction or injection process. The time derivative of the displacement vector is denoted by $\dot{u}$. Results on the existence and uniqueness of the solution for these models have been investigated by Showalter in [4] and by Zenisek in [5], and the well-posedness for nonlinear poroelastic models is considered, for example, in [6].

Biot's models are still used today in a great variety of fields, ranging from geomechanics and petroleum engineering, where these models have been applied ever since their discovery, to biomechanics or even food processing more recently. Some examples of applications in geosciences include petroleum production, solid waste disposal, carbon sequestration, soil consolidation, glaciers dynamics, subsidence, liquefaction and hydraulic fracturing, for instance. In biomechanics the poroelastic theory can be used to describe tumor-induced stresses in the brain (see [7]), which can cause deformation of the surrounding tissue, and bone deformation under a mechanical load (see [8]), for example. More recently, a promising and innovative application studies the food processes as a multiphase deformable porous media, in order to improve the quality and safety of the food, see [9].

Although some analytical solutions have been derived for some linear poroelasticity problems, see [10], and even some of them are obtained artificially as in [11], numerical simulations seem to be the only way to obtain quantitative results for real applications. The numerical solution of these problems is usually based on finite element methods, see for example the monograph of Lewis and Schrefler in [12] and the papers in [13, 14, 15, 16]. Finite difference methods have been also applied to solve this problem, see for example the convergence analysis in [17] and the extension to the discontinuous coefficients case in $[18,19]$. 
It is well-known that approximations by standard finite difference and finite element methods of the poroelasticity equations often exhibit strong nonphysical oscillations in the fluid pressure, see for instance [20, 21, 22, 23, 24]. For example, this is the case when linear finite elements are used to approximate both displacement and pressure unknowns, or when a central finite difference scheme on collocated grids is considered. To eliminate such instabilities, approximation spaces for the vector and scalar fields, satisfying an appropriate inf-sup condition (see [25]) are commonly used. Such discretizations have been theoretically investigated by Murad et al. in [26, 27, 28]. However, as seen in [29] by considering the Taylor-Hood elements [30] (approximating the displacement by continuous piecewise quadratic functions and the pressure by continuous piecewise linear functions) and as we show later in the numerical experiments by using MINI element, an inf-sup stable pair of spaces does not necessarily provide oscillation-free solutions. On the other hand, the oscillations disappear on very fine grids, when some stability restrictions between the space and time discretization parameters are fulfilled (see $[29,31]$ ), but evidently, this is not always practical.

Our work here is on investigating mechanisms for avoiding the nonphysical oscillations in the discrete solution, for example, by adding stabilization terms to the Galerkin formulation, while still maintaining the accuracy of approximations. Such strategy has been applied in [29] to provide a stable scheme by using linear finite element approximations for both unknowns. This was accomplished by adding an artificial term, namely, the time derivative of a diffusion operator multiplied by a stabilization parameter, to the flow equation. The stabilization parameter, which depends on the elastic properties of the solid and on the characteristic mesh size, was given a priori, and its optimality was shown in the one-dimensional case. This scheme provided solutions without oscillations independently of the chosen discretization parameters. Following the same strategy as in [29] for P1-P1 elements, we can provide a stabilized scheme from the classical MINI element. In the onedimensional case, we can deduce which is the exact stabilization parameter in order to transform the discrete decoupled pressure equation into the standard discretization of the heat-type equation appearing after decoupling the continuous system. It results that adding the following term,

$$
\varepsilon \frac{h^{2}}{\lambda+2 \mu} \int_{\Omega}\left(\frac{\nabla p_{h}^{m+1}-\nabla p_{h}^{m}}{\tau}\right) \cdot \nabla q_{h} \mathrm{~d} x
$$

to the second equation in the discrete variational formulation of problem (1)- 
(2), where $\varepsilon=1 / 4$ for the linear finite element pair and $\varepsilon=1 / 6$ for the MINI element method, we provide solutions without non-physical oscillations independently on the discretization parameters. This is rigorous in the one-dimensional case, while in the multidimensional case we extend straightforwardly this strategy without having a proof of the monotonicity of the resulting schemes. However, in the numerical experiments we will see that in all cases the use of these stabilization parameters completely remove the spurious oscillations.

In this work, we present convergence analysis of fully discrete implicit schemes for the numerical solution of Biot's consolidation model by using both stabilized MINI element and P1-P1 discretizations, and numerically show that such choices of stabilization parameters and operators remove the non-physical oscillations in the approximations of the pressure. In this regard, our work fills in a gap in the literature, since to our knowledge the results presented here are the first theoretical results for fully discrete schemes involving stabilized spatial discretizations aimed to improve the monotonicity properties of the finite element schemes.

The rest of the paper is organized as follows. In Section 2 we show several abstract results on stabilized discretizations which we use in Section 3 to analyze the convergence of the fully discrete model. The abstract results in Section 2 apply to more general saddle-point problems with stabilization terms. In this section, we have also computed the exact Schur complement corresponding to the bubble functions in the MINI element. Next, in Section 3 we use the abstract results and show first order convergence in time and space for the fully discrete Biot's consolidation model. The Section 4 is devoted to the numerical study of the convergence and monotonicity properties of the resulting discretizations. We use several benchmark tests in poromechanics and show that appropriate choice of stabilization parameters result in approximations which respect the underlying physical behavior and are oscillation-free. Conclusions are drawn in Section 5.

\section{Stability of discretizations and perturbations of Biot's model}

In this section we provide results on the stability of discretizations of saddle point problems that can be viewed as perturbations of the Stokes equations. By stability, here, we mean bounds on the inverse of the discrete operator (for a fixed time step). We prove inf-sup condition for different discretizations for the poroelasticity problem, more precisely for MINI ele- 
ment and stabilized P1-P1 schemes. Such results are well-known for Stokes equations (see, e.g. [32, 33, 34]).

We hope that the results given below in Section 2.1 will be useful in other situations. We note that the generality of the abstract results allows us to use an unweighted $L^{2}$ norm for the pressure (not only an energy norm), which gives new estimates in the analysis of the fully discretized time dependent Biot's model.

\subsection{Stability of a class of saddle point problems with perturbation}

In this section, we consider operators of the form

$$
\mathcal{A}_{C}=\left(\begin{array}{cc}
A & B^{\prime} \\
B & -C
\end{array}\right): V \times Q \mapsto V^{\prime} \times Q^{\prime},
$$

where $V$ and $Q$ are Hilbert spaces and $V^{\prime}$ and $Q^{\prime}$ are their dual spaces. Here, $\langle\cdot, \cdot\rangle$ is the standard duality pairing and $B^{\prime}: Q \mapsto V^{\prime}$ is the adjoint of $B$. We make the following assumptions on $A$ and $C$.

(A1) The operator $A: V \mapsto V^{\prime}$ is bounded, selfadjoint and positive definite. Thus, $A$ provides a scalar product $(\cdot, \cdot)_{A}=\langle A \cdot, \cdot\rangle$ and a norm on $V$ denoted by $\|\cdot\|_{A}$. The Hilbert Space $V$ is then equipped with this inner product and norm, and we have that

$$
\begin{aligned}
& \|v\|_{A}^{2}:=\langle A v, v\rangle, \quad\|f\|_{V^{\prime}}^{2}:=\left\langle f, A^{-1} f\right\rangle, \quad \text { for all } \quad v \in V, \quad f \in V^{\prime} \\
& \|A\|_{V \mapsto V^{\prime}}=\left\|A^{-1}\right\|_{V^{\prime} \mapsto V}=1 .
\end{aligned}
$$

(A2) The operator $B: V \mapsto Q^{\prime}$ is bounded.

(A3) Similarly to $A$, the operator $C: Q \mapsto Q^{\prime}$ is bounded, selfadjoint and positive (semi)definite. Thus on $Q$ we have a norm (or a semi-norm) denoted by $\|\cdot\|_{C}$

We introduce a norm on $V \times Q$ :

$$
\|(u, p)\|^{2}=\|u\|_{A}^{2}+\|p\|_{C}^{2}+\|p\|^{2} .
$$

We note that if $C$ is only semidefinite, then $\|\cdot\|_{C}$ is only a seminorm on $Q$. Here $\|\cdot\|$ denotes the norm on $Q$ and $\|\cdot\|$ is the norm on $V \times Q$ in which we will prove stability estimates for the operator $\mathcal{A}_{C}$.

Clearly, $\mathcal{A}_{C}$ can be viewed as a perturbation of $\mathcal{A}_{0}$, i.e. the operator with $C=0$. For the error analysis, we need a necessary and sufficient condition 
for $\mathcal{A}_{C}$ to be isomorphism under the assumptions (A1)-(A3). Although more general results also hold (with $A$ and $C$ only invertible on subspaces), to prove them would require more elaborate arguments and such generality is beyond the scope of our considerations here. Proofs, in the most general case, are found in a paper by Zulehner [35] on nonstandard norms associated with general saddle point problems. Other results on stability of such perturbed operators are found in a monograph by Boffi, Brezzi and Fortin [34] (see also [36]).

We have the following theorem when assumptions (A1)-(A3) hold.

Theorem 1. Assume that (A1)-(A3) hold. Then $\mathcal{A}_{C}$ defined in (4) is an isomorphism if and only if the operator $B$ satisfies the following inf-sup condition: For any $q \in Q$ we have

$$
\sup _{v \in V} \frac{\langle B v, q\rangle}{\|v\|_{A}} \geq \gamma_{B}\|q\|-\|q\|_{C}
$$

Proof. We first assume that (6) holds and we introduce the bilinear form

$$
\left\langle\mathcal{A}_{C}(u, p) ;(v, q)\right\rangle=\langle A u, v\rangle+\langle B v, p\rangle+\langle B u, q\rangle-\langle C p, q\rangle .
$$

It is easy to verify that the operator $\mathcal{A}_{C}$ is bounded in $\|\cdot\|$ since both $A$ and $B$ are continuous. From the inf-sup condition (6), for any $p$, there exist $w \in V$, such that $\langle B w, p\rangle \geq\left(\gamma_{B}\|p\|-\|p\|_{C}\right)\|w\|_{A}$. Since this inequality does not change when we multiply $w$ by a positive scalar, without loss of generality, we may assume that $\|w\|_{A}=\|p\|$. We then have,

$$
\langle B w, p\rangle \geq\left(\gamma_{B}\|p\|-\|p\|_{C}\right)\|p\| \text {. }
$$

For a given pair $(u, p) \in V \times Q$ and with $w$ defined as above, we choose $v=u+\theta w$, and, $q=-p$, with some $\theta>0$ to be determined later. Using the inf-sup condition, the fact that $\|w\|_{A}=\|p\|$ and applying some obvious inequalities, such as, $a b \geq-\frac{1}{2 \theta} a^{2}-\frac{\theta}{2} b^{2}$, we have

$$
\begin{aligned}
\left\langle\mathcal{A}_{C}(u, p) ;(v, q)\right\rangle & =\langle A u, u+\theta w\rangle+\langle B(u+\theta w), p\rangle-\langle B u, p\rangle+\langle C p, p\rangle \\
& =\|u\|_{A}^{2}+\theta\langle A u, w\rangle+\theta\langle B w, p\rangle+\|p\|_{C}^{2} \\
& \geq \frac{1}{2}\|u\|_{A}^{2}-\frac{\theta^{2}}{2}\|p\|^{2}+\theta \gamma_{B}\|p\|^{2}-\theta\|p\|_{C}\|p\|+\|p\|_{C}^{2} \\
& \geq \frac{1}{2}\|u\|_{A}^{2}+\left(\theta \gamma_{B}-\frac{\theta^{2}}{2}\right)\|p\|^{2}-\theta\left(\frac{1}{2 \theta}\|p\|_{C}^{2}+\frac{\theta}{2}\|p\|^{2}\right)+\|p\|_{C}^{2} .
\end{aligned}
$$


Since the inequality above holds for any $\theta>0$, we choose $\theta=\frac{\gamma_{B}}{2}$ to obtain that

$$
\left\langle\mathcal{A}_{C}(u, p) ;(v, q)\right\rangle \geq \frac{1}{2}\|u\|_{A}^{2}+\frac{\gamma_{B}^{2}}{4}\|p\|^{2}+\frac{1}{2}\|p\|_{C}^{2} \geq \widetilde{\gamma}\|(u, p)\|^{2}
$$

where $\widetilde{\gamma}=\frac{1}{4} \min \left\{2, \gamma_{B}^{2}\right\}$. On the other hand, the triangle inequality implies that

$$
\|(v, q)\|=\|(u+\theta w, p)\| \leq \widetilde{\gamma}_{1}\|(u, p)\|,
$$

with $\widetilde{\gamma}_{1}$ depending only on $\gamma_{B}$. Hence,

$$
\sup _{v, q} \frac{\left\langle\mathcal{A}_{C}(u, p) ;(v, q)\right\rangle}{\|(v, q)\|} \geq \gamma\|(u, p)\|, \quad \gamma=\frac{\widetilde{\gamma}}{\widetilde{\gamma}_{1}}
$$

which shows that $\mathcal{A}_{C}$ is an isomorphism.

To prove the other direction, that the invertibility of $\mathcal{A}_{C}$ implies condition (6), for any $q \in Q$, we define $v_{q}=-A^{-1} B^{\prime} q \in V$. Since $\mathcal{A}_{C}\left(\begin{array}{c}v_{q} \\ q\end{array}\right)=$ $\left(\begin{array}{c}0 \\ B v_{q}-C q\end{array}\right)$ the invertibility of $\mathcal{A}_{C}$ implies that

$$
\|q\| \leq\left\|\left(v_{q}, q\right)\right\| \leq\left\|\mathcal{A}_{C}^{-1}\right\|\left\|B v_{q}-C q\right\|_{Q^{\prime}} \leq\left\|\mathcal{A}_{C}^{-1}\right\|\left(\left\|B v_{q}\right\|_{Q^{\prime}}+\|C q\|_{Q^{\prime}}\right) .
$$

Since $C$ is symmetric and positive (semi)-definite, we have $\langle C q, s\rangle \leq \sqrt{\langle C q, q\rangle} \sqrt{\langle C s, s\rangle}$. Hence,

$$
\|C q\|_{Q^{\prime}}=\sup _{s \in Q} \frac{\langle C q, s\rangle}{\|s\|} \leq \sqrt{\|C\|\langle C q, q\rangle}
$$

To estimate $\left\|B v_{q}\right\|_{Q^{\prime}}$ we observe that $\left\|B v_{q}\right\|_{Q^{\prime}}=\sup _{s \in Q} \frac{\left\langle B v_{q}, s\right\rangle}{\|s\|}$ and we also have for all $s \in Q$,

$$
\begin{aligned}
\frac{\left|\left\langle B v_{q}, s\right\rangle\right|}{\|s\|} & =\frac{\left|\left\langle B^{\prime} s, A^{-1} B^{\prime} q\right\rangle\right|}{\|s\|} \leq\left\|B^{\prime}\right\| \frac{\left|\left\langle B^{\prime} s, A^{-1} B^{\prime} q\right\rangle\right|}{\left\|B^{\prime} s\right\|_{V^{\prime}}} \\
& \leq\left\|B^{\prime}\right\| \sup _{f \in V^{\prime}} \frac{\left\langle f, A^{-1} B^{\prime} q\right\rangle}{\|f\|_{V^{\prime}}}=\left\|B^{\prime}\right\| \sup _{w \in V} \frac{\left\langle A w, A^{-1} B^{\prime} q\right\rangle}{\|A w\|_{V^{\prime}}} \\
& \leq\left\|B^{\prime}\right\|\left\|A^{-1}\right\| \sup _{w \in V} \frac{\langle B w, q\rangle}{\|w\|_{A}}=\left\|B^{\prime}\right\| \sup _{w \in V} \frac{\langle B w, q\rangle}{\|w\|_{A}} .
\end{aligned}
$$

The inf-sup condition (6) easily follows by combining the last two estimates. 
We have the following immediate corollaries.

Corollary 1. Suppose that (A1)-(A3) hold. If $\mathcal{A}_{0}$ is an isomorphism, then $\mathcal{A}_{C}$ is an isomorphism for all continuous and positive (semi-)definite $C$.

Proof. From the fact that $\mathcal{A}_{0}$ is isomorphism it follows that (6) holds with $C=0$, and hence, also with any symmetric positive, (semi-)definite and bounded $C$. This in turn (by Theorem 1 ) implies that $\mathcal{A}_{C}$ is an isomorphism.

The next corollary allows us to add consistent perturbations to already stable discretizations in order to improve the monotonicity properties of the underlying discretizations.

Corollary 2. Suppose that $\mathcal{A}_{C}$ is an isomorphism, that (A1)-(A3) hold, and that $D$ is spectrally equivalent to $C$, namely $\alpha_{0}\|q\|_{C} \leq\|q\|_{D} \leq \alpha_{1}\|q\|_{C}$ for some positive constants $\alpha_{0}$ and $\alpha_{1}$. Then $\mathcal{A}_{D}$ is an isomorphism.

Proof. For all $q \in Q$, we have

$\|q\|_{D}+\sup _{v \in V} \frac{\langle B v, q\rangle}{\|v\|_{A}} \geq \min \left\{1, \alpha_{0}\right\}\left(\|q\|_{C}+\sup _{v \in V} \frac{\langle B v, q\rangle}{\|v\|_{A}}\right) \geq \min \left\{1, \alpha_{0}\right\} \gamma_{B}\|q\|$,

which shows (6) for $\mathcal{A}_{D}$. Applying Theorem 1 gives the desired result.

\subsection{Application to discretizations of Biot's model}

After a time discretization (backward Euler scheme in time) of the Biot's model, the following system of differential equations is solved on every time step on a domain $\Omega \subset \mathbb{R}^{d}$ :

$$
\begin{aligned}
& -\operatorname{div} \sigma+\nabla p=f, \quad \sigma=2 \mu \varepsilon(u)+\lambda \operatorname{div}(u) I \\
& -\operatorname{div} u+\tau \operatorname{div} K \nabla p=g .
\end{aligned}
$$

A typical set of boundary conditions is

$$
\begin{aligned}
& u=0, \quad \text { and }(K \nabla p \cdot n)=0, \quad \text { on } \Gamma_{c}, \\
& \sigma \cdot n=\beta, \quad \text { and } p=0 \text { on } \Gamma_{t} .
\end{aligned}
$$

To introduce the spatial discretization of the Biot's model, we consider finite dimensional spaces $V_{h} \subset\left[H_{\Gamma_{c}}^{1}(\Omega)\right]^{d}$ and $Q_{h} \subset H_{\Gamma_{t}}^{1}(\Omega)$ where $H_{\Gamma_{c}}^{1}(\Omega)$ 
and $H_{\Gamma_{t}}^{1}(\Omega)$ are the standard Sobolev spaces with functions whose traces vanish on $\Gamma_{c}$ and $\Gamma_{t}$ respectively.

We have the following discrete formulation (on each time step) corresponding to $(7)-(8)$. Find $(u, p) \in V_{h} \times Q_{h}$ such that

$$
\begin{aligned}
& a(u, v)-(\operatorname{div} v, p)=(f, v), \quad \text { for all } \quad v \in V_{h}, \\
& -(\operatorname{div} u, q)-\tau a_{p}(p, q)=(g, q), \quad \text { for all } \quad q \in Q_{h} .
\end{aligned}
$$

The bilinear forms $a(\cdot, \cdot)$ and $a_{p}(\cdot, \cdot)$ are as follows:

$$
a(u, v)=2 \mu \int_{\Omega} \varepsilon(u): \varepsilon(v)+\lambda \int_{\Omega} \operatorname{div} u \operatorname{div} v, \quad a_{p}(p, q)=\int_{\Omega} K \nabla p \cdot \nabla q .
$$

The corresponding operators $A: V_{h} \mapsto V_{h}^{\prime}, B: Q_{h} \mapsto V_{h}^{\prime}$, and the norm on $Q,\|\cdot\|$, are defined as follows:

$$
\begin{aligned}
& \langle A u, v\rangle:=a(u, v), \quad\langle B u, q\rangle:=-(\operatorname{div} u, q), \quad\left\langle A_{p} p, q\right\rangle:=a_{p}(p, q), \\
& \|q\|^{2}:=\tau\left\langle A_{p} q, q\right\rangle+\|q\|_{L^{2}(\Omega)}^{2} .
\end{aligned}
$$

Since $C$ may take different form for different discretizations, we do not specify its definition here.

\subsubsection{Discretization with MINI element}

We consider a discretization with MINI element, introduced in [32] where the finite element spaces that we use are as follows:

$$
V_{h} \times Q_{h}, \quad \text { where } \quad V_{h}=V_{l} \oplus V_{b},
$$

where $V_{l}$ is the space of piece-wise (with respect to a triangulation $\mathcal{T}_{h}$ ) linear continuous vector valued functions on $\Omega$ and $V_{b}$ is the space of bubble functions, defined as

$$
V_{b}=\operatorname{span}\left\{\varphi_{b, T} e_{1}, \ldots, \varphi_{b, T} e_{d}\right\}_{T \in \mathcal{T}_{h}}, \quad \varphi_{b, T}=\alpha_{T} \lambda_{1, T} \ldots \lambda_{d+1, T},
$$

where $\lambda_{m, T}$ are the barycentric coordinates on $T, e_{j}$ are the canonical Euclidean basis vectors in $\mathbb{R}^{d}$ and $\alpha_{T}$ is a normalizing constant for $\varphi_{b, T}$. The function $\varphi_{b, T}$ is scalar valued and is called a bubble function. The space $Q_{h}$ consists of piece-wise linear continuous scalar valued functions.

Note that if we write $v=v_{l}+v_{b}$ we have that

$$
a(u, v)=a\left(u_{l}, v_{l}\right)+a\left(u_{b}, v_{b}\right) .
$$


This is so because $v_{b}$ is zero on $\partial T$ for $T \in T_{h}$ and integration by parts shows that $a\left(v_{l}, v_{b}\right)=0$. We then have the following block form of the discrete problem (9)-(10):

$$
\mathcal{A}\left(\begin{array}{l}
u_{b} \\
u_{l} \\
p
\end{array}\right)=\left(\begin{array}{l}
f_{b} \\
f_{l} \\
g
\end{array}\right), \quad \text { where } \mathcal{A}=\left(\begin{array}{ccc}
A_{b} & 0 & G_{b} \\
0 & A_{l} & G_{l} \\
G_{b}^{T} & G_{l}^{T} & -\tau A_{p}
\end{array}\right)
$$

The operators $A_{b}, A_{l}, G_{b}, G_{l}$ and $A_{p}$ correspond to the following bilinear forms:

$$
\begin{aligned}
& a\left(u_{b}, v_{b}\right) \rightarrow A_{b}, \quad a\left(u_{l}, v_{l}\right) \rightarrow A_{l}, \quad(K \nabla p, \nabla q) \rightarrow A_{p} \\
& -\left(\operatorname{div} v_{b}, p\right)=\left(v_{b}, \nabla p\right) \rightarrow G_{b}, \quad-\left(\operatorname{div} v_{l}, p\right) \rightarrow G_{l}, \\
& u_{b}, v_{b} \in V_{b}, \quad u_{l}, v_{l} \in V_{l}, \quad p, q \in Q_{h} .
\end{aligned}
$$

It is well known that inf-sup condition holds for the MINI element for the Stokes problem, and therefore, by Corollary 1, we obtain the following inf-sup condition for MINI element discretization of poro-elasticity operator: There exists $\gamma_{0}$ independent of $h, \tau$ and $K$, such that for any $(v, q) \in V_{h} \times Q_{h}$ we have

$$
\sup _{(w, s) \in V_{h} \times Q_{h}} \frac{(\mathcal{A}(v, q),(w, s))}{\|(w, s)\|} \geq \gamma_{0}\|(v, q)\| .
$$

As it is well-known (see [25]), equation (12) is equivalent to the estimate

$$
\|(u, p)\| \leq \gamma_{0}^{-1}\|(f, g)\| .
$$

\subsection{Stabilization via elimination of bubbles}

All P1-P1 stabilized discretizations which we consider here, are derived from the MINI element by eliminating locally the bubble functions. For details on such stabilizations we refer to the classical paper by Brezzi and Pitkäranta [37] and also to a work by P. Roger [38].

We now consider the following operator on $V_{l} \times Q_{h}$ :

$$
\mathcal{A}_{l}=\left(\begin{array}{cc}
A_{l} & G_{l} \\
G_{l}^{T} & -\left(\tau A_{p}+S_{b}\right)
\end{array}\right), \quad \text { where } \quad S_{b}=G_{b}^{T} A_{b}^{-1} G_{b}
$$

which is obtained after eliminating the equation corresponding to bubble functions from (11). This is also an operator of the form given in (4) with $C=\tau A_{p}+S_{b}$. We have the following theorem: 
Theorem 2. Suppose that the triple $\left(u_{b}, u_{l}, p\right)$ solves

$$
\mathcal{A}\left(\begin{array}{l}
u_{b} \\
u_{l} \\
p
\end{array}\right)=\left(\begin{array}{l}
0 \\
f_{l} \\
g
\end{array}\right) \text {. }
$$

Then the pair $\left(u_{l}, p\right)$ solves

$$
\mathcal{A}_{l}\left(\begin{array}{c}
u_{l} \\
p
\end{array}\right)=\left(\begin{array}{l}
f_{l} \\
g
\end{array}\right) .
$$

Moreover, a uniform inf-sup condition such as (12) holds: For any $\left(v_{l}, q\right) \in$ $V_{l} \times Q_{h}$,

$$
\sup _{\left(w_{l}, s\right) \in V_{l} \times Q_{h}} \frac{\left(\mathcal{A}_{l}\left(v_{l}, q\right),\left(w_{l}, s\right)\right)}{\left\|\left(w_{l}, s\right)\right\|} \geq \gamma_{1}\left\|\left(v_{l}, q\right)\right\| .
$$

Proof. Since $\left(u_{b}, u_{l}, p\right)$ solves the system (14) we have that

$$
\begin{aligned}
& u_{b}=-A_{b}^{-1} G_{b} p \\
& A_{l} u_{l}+G_{l} p=f_{l} . \\
& G_{l}^{T} u_{l}+G_{b}^{T} u_{b}-\tau A_{p} p=g \Longrightarrow G_{l}^{T} u_{l}-\left(G_{b}^{T} A_{b}^{-1} G_{b}+\tau A_{p}\right) p=g .
\end{aligned}
$$

From this we conclude that $\left(u_{l}, p\right)$ solves $(15)$. Now, since $\left(u_{b}, u_{l}, p\right)$ solves $(14)$, from (13),

$$
\left\|\left(u_{b}, u_{l}, p\right)\right\| \leq \gamma_{0}^{-1}\left\|\left(0, f_{l}, g\right)\right\|,
$$

and therefore we have

$$
\left\|\left(u_{l}, p\right)\right\| \leq\left\|\left(u_{b}, u_{l}, p\right)\right\| \leq \gamma_{0}^{-1}\left\|\left(0, f_{l}, g\right)\right\|=\gamma_{0}^{-1}\left\|\left(f_{l}, g\right)\right\| .
$$

This estimate shows that $\mathcal{A}_{l}$ is a bounded isomorphism, which is equivalent to the inf-sup condition (16). This completes the proof.

Applying Corollary 2 to $\mathcal{A}_{l}$ then shows that any operator $C: Q_{h} \mapsto Q_{h}^{\prime}$, spectrally equivalent to $\tau A_{p}+S_{b}$ will result in a stable discretization of the Biot's model. As we show in the next section (Theorem 3), the perturbations spectrally equivalent to $S_{b}$ are of the form

$$
\langle C p, q\rangle=\sum_{T \in \mathcal{T}_{h}} C_{T} h_{T}^{2} \int_{T}(\nabla p \cdot \nabla q),
$$

where $C_{T}, T \in \mathcal{T}_{h}$ are constants independent of the mesh size $h$ or $\tau$. 


\subsection{Perturbations, spectrally equivalent to the Schur complement}

In this section we discuss the exact Schur complement (the perturbation or the stabilization) given by $S_{b}=G_{b}^{T} A_{b}^{-1} G_{b}$. We denote $V_{b, T}=\operatorname{span} \varphi_{b, T}$ and we have that $V_{b}=\oplus_{T \in \mathcal{T}_{h}} V_{b, T}$. Let $n_{V}$ be the number of vertices in the triangulation, $n_{T}$ be the number of elements, and $n_{b}=d n_{T}$. Note that $n_{b}$ equals the dimension of $V_{b}$. With every element $T \in \mathcal{T}_{h}$ we associate the incidence matrices $I_{T} \in \mathbb{R}^{n_{V} \times(d+1)}$ and $J_{T} \in \mathbb{R}^{n_{b} \times d}$ mapping the local degrees of freedom on $T$ to the degrees of freedom corresponding to $Q$ and $V_{b}$.

Let us now give a more precise definition of the incidence matrices $I_{T}$ and $J_{T}$ for an element $T \in \mathcal{T}_{h}$, with vertices $\left(j_{1}, \ldots, j_{d+1}\right), j_{k} \in\left\{1, \ldots, n_{V}\right\}$, and $j_{\ell} \neq j_{m}$, for $j \neq m$. Let $\left\{\delta_{1}, \ldots, \delta_{d+1}\right\},\left\{e_{1}, \ldots, e_{n_{V}}\right\},\left\{f_{1}, \ldots, f_{n_{b}}\right\}$ and $\left\{\eta_{1}, \ldots, \eta_{d}\right\}$ be the canonical Euclidean bases in $\mathbb{R}^{d+1}, \mathbb{R}^{n_{V}}, \mathbb{R}^{n_{b}}$ and $\mathbb{R}^{d}$, respectively. We also denote by $\left(k_{1}, \ldots, k_{d}\right)$ the degrees of freedom corresponding to the bubble functions associated with $T \in \mathcal{T}_{h}$. We then define

$$
\mathbb{R}^{n_{V} \times(d+1)} \ni I_{T}=\sum_{m=1}^{d+1} e_{j_{m}} \delta_{m}^{T}, \quad \mathbb{R}^{n_{b} \times d} \ni J_{T}=\sum_{m=1}^{d} f_{k_{m}} \eta_{m}^{T} .
$$

Since the sets of degrees of freedom corresponding to the bubble functions in different elements do not intersect, we have $J_{T}^{T} J_{T}=I_{d \times d}$, and, $J_{T^{\prime}}^{T} J_{T}=$ 0 when $T^{\prime} \neq T$. Here $I_{d \times d} \in \mathbb{R}^{d \times d}$ is the identity matrix. Using these definitions, we easily find that

$$
\begin{aligned}
A_{b} & =\sum_{T \in \mathcal{T}_{h}} J_{T} A_{b, T} J_{T}^{T}, \quad A_{b}^{-1}=\sum_{T \in \mathcal{T}_{h}} J_{T} A_{b, T}^{-1} J_{T}^{T}, \\
G_{b} & =\sum_{T \in \mathcal{T}_{h}} J_{T} G_{b, T} I_{T}^{T} .
\end{aligned}
$$

These identities then give,

$$
S_{b}=G_{b}^{T} A_{b}^{-1} G_{b}, \quad \text { and hence } \quad S_{b}=\sum_{T \in \mathcal{T}_{h}} I_{T} G_{b, T}^{T} A_{b, T}^{-1} G_{b, T} I_{T}^{T} .
$$

We next state a spectral equivalence result which shows that $S_{b}$ introduces a stabilization term of certain order in $h$ for P1-P1 discretization. Such stabilization techniques have been discussed by several authors (see P. Roger [38], Verfürth in [39], and also $\S 8.5 .2$ and $\S 8.13 .2$ in [34]). 
Theorem 3. Let $L$ be the stiffness matrix corresponding to the Laplace operator discretized with piece-wise linear continuous finite elements. Then the following spectral equivalence result holds

$$
S_{b} \approx h^{2} L,
$$

where the constants hidden in " $\sim$ " are independent of the mesh size.

Proof. The spectral equivalence is a direct consequence from Lemma 11 in [40] and the relations given in (18).

The proof of the next result follows easily by computing exactly the local Schur complements $S_{b, T}, T \in \mathcal{T}_{h}$. Details on the proof are found in [40].

Theorem 4. The local Schur complement $S_{b, T}$ is an M-matrix whenever the local stiffness matrix on $T \in \mathcal{T}_{h}$ corresponding to the Laplace operator discretized with piece-wise linear continuous finite elements is an M-matrix.

The spectral equivalence in Theorem 3 and the analysis that follows justifies the addition of stabilization terms to both the MINI element and the stabilized P1-P1 discretizations. These results also hold for one, two and three spatial dimensions and also give the exact perturbation (stabilization) to P1-P1 elements that provides inf-sup condition with the same constant as the MINI element See the Appendix in [40] for the detailed calculations.

Related results (in 2D) are found in a paper on Stokes equations by Bank and Welfert [41] where it was shown that in 2D the elimination of the bubbles in the MINI element gives the Petrov-Galerkin discretization by Hughes, Franka and Balestra [42] and Brezzi and Douglas [43]. Here we not only compute the exact Schur complement in any spatial dimension, but we also show that the perturbation is spectrally equivalent to a scaling of the discretization of the Laplacian with piece-wise linear finite elements and is an $M$-matrix in case the local matrices for the Laplace equation are $M$-matrices. The details and the proofs of Theorem 3 and Theorem 4 are somewhat elaborate and the interested reader can find all details in [40, Appendix].

Such results, however, do not say anything about the monotonicity of the corresponding discretization (except in 1D, where a further stabilization can be introduced in order to obtain provable monotone discrete scheme). In fact, for the one dimensional case the minimum amount of stabilization that provides monotone discretization can be calculated precisely. In general, 
even for two and three spatial dimensions, adding a stabilization term of the form $c h^{2} L$ in case when $L$ is a Stieltjes matrix improves the monotonicity properties of the resulting discrete problem. This is natural to expect because a Stieltjes matrix is monotone. Indeed, the numerical results that we present later also show that adding such stabilizations leads to monotone schemes. However, no theoretical results on the monotonicity of the discrete operators for two and three dimensional problems are available in the literature and seem to be very hard to establish.

\section{Error estimates for the fully discrete problem}

In this section, we consider the error analysis of the finite element discretization of the Biot's model. To simplify the notation and without loss of generality in this section we assume that the boundary conditions for both the displacement $u$ and the pressure $p$ are homogeneous Dirichlet boundary conditions. Then, the weak form of the Biot's model is as follows: Find $u(t) \in\left[H_{0}^{1}(\Omega)\right]^{d}$ and $p(t) \in H_{0}^{1}(\Omega)$, such that

$$
\begin{array}{ll}
a(u, v)-(\operatorname{div} v, p)=(f, v), & \forall v \in\left[H_{0}^{1}(\Omega)\right]^{d}, \\
-\left(\operatorname{div} \partial_{t} u, q\right)-a_{p}(p, q)=0, & \forall q \in H_{0}^{1}(\Omega),
\end{array}
$$

with the initial data $u(0)$ and $p(0)$ given by the solution of the following Stokes problem: Find $u(0) \in\left[H_{0}^{1}(\Omega)\right]^{d}$ and $p(0) \in L^{2}(\Omega)$, such that,

$$
\begin{aligned}
& a(u(0), v)-(\operatorname{div} v, p(0))=(f(0), v), \quad \forall v \in\left[H_{0}^{1}(\Omega)\right]^{d}, \\
& -(\operatorname{div} u(0), q)=0, \quad \forall q \in L^{2}(\Omega),
\end{aligned}
$$

We consider the fully discretized scheme at time $t_{n}, n=1,2, \ldots$, as the following: Find $u_{h}^{n}=u_{h}\left(t_{n}\right) \in V_{h} \subset\left[H_{0}^{1}(\Omega)\right]^{d}$ and $p_{h}^{n}=p_{h}\left(t_{n}\right) \in Q_{h} \subset$ $H_{0}^{1}(\Omega)$, such that,

$$
\begin{aligned}
& a\left(u_{h}^{n}, v_{h}\right)-\left(\operatorname{div} v_{h}, p_{h}^{n}\right)=\left(f\left(t_{n}\right), v_{h}\right), \quad \forall v_{h} \in V_{h}, \\
& -\left(\operatorname{div} \bar{\partial}_{t} u_{h}^{n}, q_{h}\right)-a_{p}\left(p_{h}^{n}, q_{h}\right)-\varepsilon h^{2}\left(\nabla \bar{\partial}_{t} p_{h}^{n}, \nabla q_{h}\right)=0, \quad \forall q_{h} \in Q_{h},
\end{aligned}
$$

where $\bar{\partial}_{t} u_{h}^{n}:=\left(u_{h}^{n}-u_{h}^{n-1}\right) / \tau$ and $\bar{\partial}_{t} p_{h}^{n}:=\left(p_{h}^{n}-p_{h}^{n-1}\right) / \tau$. Here we try to analyze MINI element and stabilized P1-P1 element in a unified way, therefore, the finite element spaces $V_{h}$ and $Q_{h}$ denote both Stokes pairs. We also define the following norm on the finite element spaces:

$$
\|(u, p)\|_{\tau, h}:=\left(\|u\|_{a}^{2}+\tau\|p\|_{a_{p}}^{2}+\varepsilon h^{2}\|\nabla p\|^{2}\right)^{1 / 2}
$$


We further denote by $\|\cdot\|_{k}$ and $|\cdot|_{k}$ the norms and seminorms in the Sobolev space $H^{k}(\Omega)$, and without loss of generality, by $\|\cdot\|$ the $L^{2}(\Omega)$ norm, i.e. $\|\cdot\|=\|\cdot\|_{0}$. Below we also denote by $c$ a generic constant independent of time step, mesh size and other important parameters.

For the initial data $u_{h}^{0}$ and $p_{h}^{0}$, we will consider two cases. First case is that they are given by the following stabilized Stokes equation:

$$
\begin{array}{ll}
a\left(u_{h}^{0}, v_{h}\right)-\left(\operatorname{div} v_{h}, p_{h}^{0}\right)=\left(f(0), v_{h}\right) & \forall v_{h} \in V_{h}, \\
-\left(\operatorname{div} u_{h}^{0}, q_{h}\right)-\varepsilon h^{2}\left(\nabla p_{h}^{0}, \nabla q_{h}\right)=0 & \forall q_{h} \in Q_{h} .
\end{array}
$$

Second case is that they do not satisfy (27) and (28) but are defined as follows,

$$
\operatorname{div} u_{h}^{0}=0 \text { and } p_{h}^{0}=0 .
$$

To derive error analysis of the fully discretized scheme (24)-(25), following the standard error analysis of time-dependent problems in Thomée [44], we first define the following elliptic projections $\bar{u}_{h}$ and $\bar{p}_{h}$ for $t>0$ as usual,

$$
\begin{aligned}
& a\left(\bar{u}_{h}, v_{h}\right)-\left(\operatorname{div} v_{h}, \bar{p}_{h}\right)=a\left(u, v_{h}\right)-\left(\operatorname{div} v_{h}, p\right), \quad \forall v_{h} \in V_{h} \\
& a_{p}\left(\bar{p}_{h}, q_{h}\right)=a_{p}\left(p, q_{h}\right), \quad \forall q_{h} \in Q_{h} .
\end{aligned}
$$

And then split the discretization error as follows.

$$
\begin{aligned}
& u(t)-u_{h}(t)=\left(u(t)-\bar{u}_{h}(t)\right)-\left(u_{h}(t)-\bar{u}_{h}(t)\right)=: \rho_{u}-e_{u} \\
& p(t)-p_{h}(t)=\left(p(t)-\bar{p}_{h}(t)\right)-\left(p_{h}(t)-\bar{p}_{h}(t)\right)=: \rho_{p}-e_{p} .
\end{aligned}
$$

For $t=t_{n}$ we use the short hand notation $\rho_{u}^{n}=\rho_{u}\left(t_{n}\right)$, and similarly $e_{u}^{n}, \rho_{p}^{n}$, $e_{p}^{n}$ denote the values of $e_{u}, \rho_{p}$ and $e_{p}$ at time $t=t_{n}$, respectively.

For the error of the elliptic projections, because we use MINI element or P1-P1 element, we have, for all $t$,

$$
\begin{aligned}
& \left\|\rho_{u}\right\|_{a} \leq c h\left(|u|_{2}+|p|_{1}\right), \\
& \left\|\rho_{p}\right\|_{1} \leq c h|p|_{2}, \quad\left\|\rho_{p}\right\|_{a_{p}} \leq c h|p|_{2} \\
& \left\|\rho_{p}\right\| \leq c h^{2}|p|_{2} .
\end{aligned}
$$

We refer to [27] for details. Since $\partial_{t} \bar{p}=\overline{\partial_{t} p}$, we have the estimates above also for $\partial_{t} \rho_{u}$ and $\partial_{t} \rho_{p}$, where on the right side of the inequalities we have norms of $\partial_{t} u$ and $\partial_{t} p$ instead of norms of $u$ and $p$ respectively.

In order to obtain the final error estimate, we need to first estimate $e_{u}$ and $e_{p}$, the error between the elliptic projection $\left\{\bar{u}_{h}\left(t_{n}\right), \bar{p}_{h}\left(t_{n}\right)\right\}$ and the 
numerical solutions $\left\{u_{h}^{n}, p_{h}^{n}\right\}$, and then the error estimates can be derived by the triangular inequality. For the sake of the simplicity, here we will only state the following theorem about the error estimates for the error $\left(u-u_{h}\right)\left(t_{n}\right)$ and $\left(p-p_{h}\right)\left(t_{n}\right)$. Please see Appendix A for the details.

We assume that for $q=1,2, \infty$ and $s=1,2$,

$$
\begin{aligned}
& u(t) \in L^{\infty}\left((0, T],\left[H_{0}^{1}(\Omega)\right]^{d}\right) \cap L^{\infty}\left((0, T],\left[H^{2}(\Omega)\right]^{d}\right), \\
& \partial_{t} u(t) \in L^{s}\left((0, T],\left[H^{2}(\Omega)\right]^{d}\right), \quad \partial_{t t} u(t) \in L^{s}\left((0, T],\left[H_{0}^{1}(\Omega)\right]^{d}\right), \\
& p(t) \in L^{\infty}\left((0, T], H_{0}^{1}(\Omega)\right) \cap L^{\infty}\left((0, T], H^{2}(\Omega)\right), \\
& \partial_{t} p(t) \in L^{q}\left((0, T], H_{0}^{1}(\Omega)\right) \cap L^{s}\left((0, T], H^{2}(\Omega)\right), \quad \partial_{t t} p(t) \in L^{s}\left((0, T], H_{0}^{1}(\Omega)\right)
\end{aligned}
$$

Theorem 5. Let $u(t)$ and $p(t)$ be the solution of (20) and (21), $u_{h}^{n}$ and $p_{h}^{n}$ be the solution of (24) and (25). For displacement $u(t)$, we have

$$
\begin{aligned}
& \left\|\left(u\left(t_{n}\right)-u_{h}^{n}, p\left(t_{n}\right)-p_{h}^{n}\right)\right\|_{\tau, h} \\
& \leq\left\|\left(e_{u}^{0}, e_{p}^{0}\right)\right\|_{\tau, h}+c\left\{\tau\left[\int_{0}^{t_{n}}\left\|\partial_{t t} u\right\|_{1} \mathrm{~d} t+\int_{0}^{t_{n}} \varepsilon^{1 / 2} h\left|\partial_{t t} p\right|_{1} \mathrm{~d} t\right]\right. \\
& \quad+h\left[\left|u\left(t_{n}\right)\right|_{2}+\left|p\left(t_{n}\right)\right|_{1}+\left(\tau^{1 / 2}+\varepsilon^{1 / 2} h\right)\left|p\left(t_{n}\right)\right|_{2}+\int_{0}^{t_{n}}\left(\left|\partial_{t} u\right|_{2}+\left|\partial_{t} p\right|_{1}\right) \mathrm{d} t\right. \\
& \left.\left.\quad+\int_{0}^{t_{n}} \varepsilon^{1 / 2} h\left|\partial_{t} p\right|_{2} \mathrm{~d} t\right]+t_{n} \max _{1 \leq j \leq n} \varepsilon^{1 / 2} h\left\|\nabla \partial_{t} p\left(t_{j}\right)\right\|\right\}
\end{aligned}
$$

For pore pressure $p(t)$, if the initial data $u_{h}^{0}$ and $p_{h}^{0}$ satisfy (27) and (28), we have,

$$
\begin{aligned}
& \left\|p\left(t_{n}\right)-p_{h}^{n}\right\|_{a_{p}} \\
& \leq\left\|e_{p}^{0}\right\|_{a_{p}}+c\left\{\tau\left[\left(\int_{0}^{t_{n}}\left\|\partial_{t t} u\right\|_{1}^{2} \mathrm{~d} t\right)^{1 / 2}+\left(\int_{0}^{t_{n}} \varepsilon h^{2}\left\|\partial_{t t} p\right\|_{1}^{2} \mathrm{~d} t\right)^{1 / 2}\right]\right. \\
& +h\left[\left|p\left(t_{n}\right)\right|_{2}+\left(\int_{0}^{t_{n}}\left(\left|\partial_{t} u\right|_{2}+\left|\partial_{t} p\right|_{1}\right)^{2} \mathrm{~d} t\right)^{1 / 2}+\left(\int_{0}^{t_{n}} \varepsilon h^{2}\left|\partial_{t} p\right|_{2}^{2} \mathrm{~d} t\right)^{1 / 2}\right] \\
& \left.\quad+\sqrt{t_{n}} \max _{1 \leq j \leq n} \varepsilon^{1 / 2} h\left\|\nabla \partial_{t} p\left(t_{j}\right)\right\|\right\} .
\end{aligned}
$$


If the initial data $u_{h}^{0}$ and $p_{h}^{0}$ are defined by (29), we have,

$$
\begin{aligned}
& \left\|p\left(t_{n}\right)-p_{h}^{n}\right\|_{a_{p}} \\
& \leq \frac{1}{\sqrt{2 \tau}}\left\|\left(e_{u}^{0}, e_{p}^{0}\right)\right\|_{\tau, h}+c\left\{\tau\left[\left(\int_{0}^{t_{n}}\left\|\partial_{t t} u\right\|_{1}^{2} \mathrm{~d} t\right)^{1 / 2}+\left(\int_{0}^{t_{n}} \varepsilon h^{2}\left\|\partial_{t t} p\right\|_{1}^{2} \mathrm{~d} t\right)^{1 / 2}\right]\right. \\
& \quad+h\left[\left|p\left(t_{n}\right)\right|_{2}+\left(\int_{0}^{t_{n}}\left(\left|\partial_{t} u\right|_{2}+\left|\partial_{t} p\right|_{1}\right)^{2} \mathrm{~d} t\right)^{1 / 2}+\left(\int_{0}^{t_{n}} \varepsilon h^{2}\left|\partial_{t} p\right|_{2}^{2} \mathrm{~d} t\right)^{1 / 2}\right] \\
& \left.\quad+\sqrt{t_{n}} \max _{1 \leq j \leq n} \varepsilon^{1 / 2} h\left\|\nabla \partial_{t} p\left(t_{j}\right)\right\|\right\} .
\end{aligned}
$$

Moreover, for pore pressure, we also have the following error estimate in $L^{2}$-norm,

$$
\begin{aligned}
& \left\|p\left(t_{n}\right)-p_{h}^{n}\right\| \\
& \leq c\left\|\left(e_{u}^{0}, e_{p}^{0}\right)\right\|_{\tau, h}+c\left\{\tau\left[\int_{0}^{t_{n}}\left\|\partial_{t t} u\right\|_{1} \mathrm{~d} t+\int_{0}^{t_{n}} \varepsilon^{1 / 2} h\left|\partial_{t t} p\right|_{1} \mathrm{~d} t\right]\right. \\
& \quad+h^{2}\left|p\left(t_{n}\right)\right|_{2}+h\left[\int_{0}^{t_{n}}\left(\left|\partial_{t} u\right|_{2}+\left|\partial_{t} p\right|_{1}\right) \mathrm{d} t+\int_{0}^{t_{n}} \varepsilon^{1 / 2} h\left|\partial_{t} p\right|_{2} \mathrm{~d} t\right] \\
& \left.\quad+t_{n} \max _{1 \leq j \leq n} \varepsilon^{1 / 2} h\left\|\nabla \partial_{t} p\left(t_{j}\right)\right\|\right\} .
\end{aligned}
$$

Remark 6. All the error estimates in Theorem 5 consist of two parts. One part is the error for $t>0$ which, in all cases, gives optimal convergence order. The other part is the error in the approximation of the initial data, i.e., $\left\|\left(e_{u}^{0}, e_{p}^{0}\right)\right\|_{\tau, h}$ and $\left\|e_{p}^{0}\right\|_{a_{p}}$. From the triangle inequality, we have

$$
\begin{aligned}
\left\|\left(e_{u}^{0}, e_{p}^{0}\right)\right\|_{\tau, h} & \leq c\left[\left\|\left(\rho_{u}^{0}, \rho_{p}^{0}\right)\right\|_{\tau, h}+\left\|\left(u(0)-u_{h}^{0}, p(0)-p_{h}^{0}\right)\right\|_{\tau, h}\right] \\
\left\|e_{p}^{0}\right\|_{a_{p}} & \leq\left\|\rho_{p}^{0}\right\|_{a_{p}}+\left\|p(0)-p_{h}^{0}\right\|_{a_{p}}
\end{aligned}
$$

where $\rho_{u}^{0}$ and $\rho_{p}^{0}$ are the errors due to the elliptic projection and $\left(u(0)-u_{h}^{0}\right)$ and $\left(p(0)-p_{h}^{0}\right)$ are the errors due to the choice of initial conditions, either satisfying Stokes equation (27) and (28) or the simpler given in (29).

If the initial data satisfies the stabilized Stokes equation (27) and (28), the initial errors strongly depend on the regularity of the initial data. A crucial role is played by the assumptions on the regularity of the pore pressure $p(0)$. If we assume $p(0) \in H_{0}^{1}(\Omega)$, then the standard error estimates for the 
elliptic projection and stabilized Stokes equation show that the initial data errors are appropriately bounded, and, hence, we have optimal order of convergence for the discrete scheme. Therefore, the overall convergence rate of the stabilized MINI element is optimal. However, if we assume that $p(0)$ is merely in $L^{2}(\Omega)$, then we cannot expect that the errors in the initial data are of optimal order, and, therefore, the overall convergence rate of the stabilized MINI element is not optimal as well.

If we just use the simple practical choice $(29)$, we cannot expect that $u_{h}^{0} p_{h}^{0}$ approximate $u(0)$ and $p(0)$ in general. Therefore, regardless of the regularity assumption of the initial data, the overall convergence rate of the stabilized MINI element will not be as desired. However, in some cases, even when the initial errors are large, they decay with respect to time (see [27]). As a consequence, the discretization error when using stabilized mini element is still optimal for sufficiently large time (long time).

\section{Numerical Experiments}

In this section, we present several numerical experiments in order to illustrate the performance of the proposed stabilized methods. We will choose well-known benchmark problems in order to deal with different aspects as variable permeability, different boundary conditions, the accuracy of the approximations, etc.

\subsection{Layered porous medium with variable permeability}

In the first experiment we want to illustrate non-monotone pressure behavior when we have a low permeability in a sub-domain. We consider a test proposed in [22] which models a porous material on which a lowpermeable layer $\left(K=10^{-8}\right)$ is placed between two layers with unit permeability $(K=1)$, as shown in Figure 4.1. The boundary of the square domain is split in two disjoint subsets $\Gamma_{1}$ and $\Gamma_{2}$ on which we assume the following boundary conditions: on the top, which is free to drain, a uniform load is applied, that is,

$$
p=0, \quad \sigma \cdot n=g, \text { with } g=(0,-1)^{t}, \text { on } \Gamma_{1},
$$

whereas at the sides and bottom that are rigid the boundary is considered to be impermeable, that is,

$$
\nabla p \cdot n=0, \quad u=0, \text { on } \Gamma_{2} .
$$




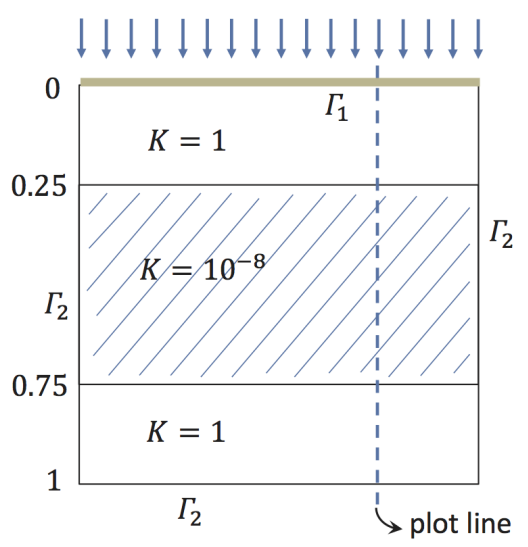

Figure 4.1: Domain representing a square of layered porous material with different permeability.

Zero initial conditions are considered for both variables, and the time step is chosen as $\tau=1$. Notice that this test can be reduced to a one-dimensional problem. Therefore, in the following simulations we will show the numerical solutions corresponding to one vertical line in the domain as displayed in Figure 4.1.

First we approximate using linear finite elements for displacements and

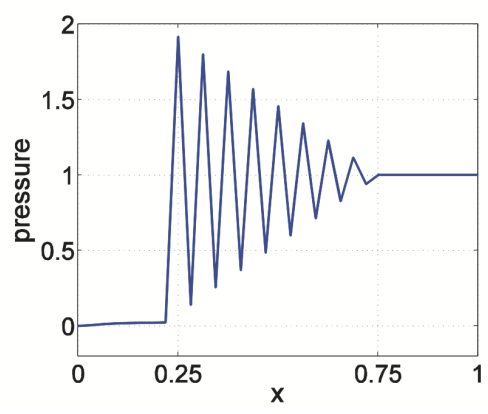

(a)

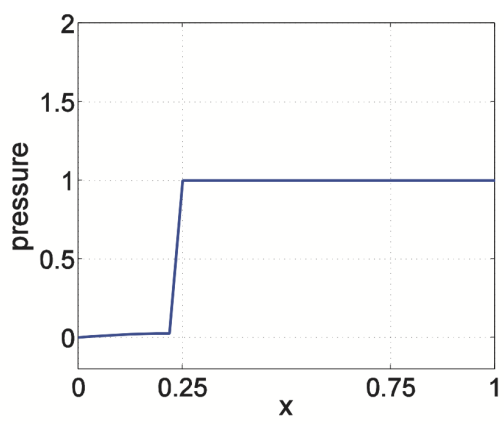

(b)

Figure 4.2: Numerical solution by P1-P1 for the pressure to the two-material problem (a) without stabilization term and (b) with stabilization term.

pressure. If no stabilization term is added to the discrete formulation, the 
approximation for the pressure field that is obtained by using 32 elements on the grid is shown in Figure 4.2 (a). We observe that strong spurious oscillations appear in the part corresponding to the low-permeable layer. However, if the stabilized scheme is used for the simulation with the same number of nodes, the oscillations are completely eliminated and the method gives rise to the monotone solution for the pressure, as we see in Figure 4.2 (b).

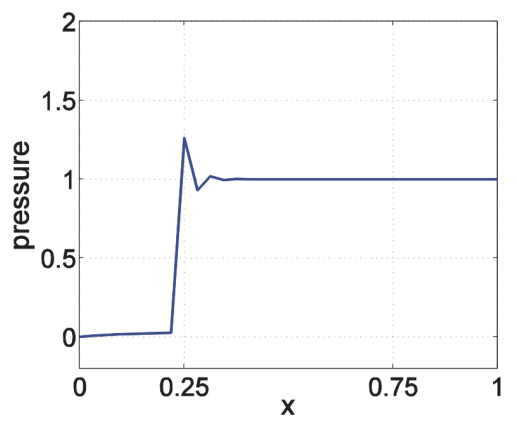

(a)

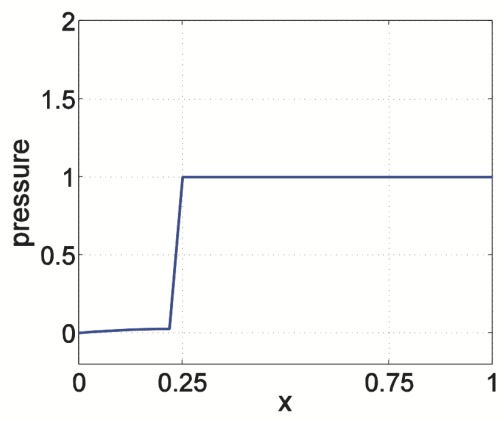

(b)

Figure 4.3: Numerical solution by P2-P1 for the pressure to the two-material problem (a) without stabilization term and (b) with stabilization term.

Next, we use approximation by MINI element with the same number of elements. Similarly to the previous case, when no stabilization parameter is included in the formulation, the oscillatory behaviour of the pressure approximation is evident, as shown in Figure 4.3 (a). Notice that the oscillations are much smaller than in the case of P1-P1 elements, but are still not eliminated by using this Stokes stable pair of spaces. Again, a perturbation stabilizes the method and we obtain oscillation-free approximation for the pressure field (see Figure 4.3 (b)).

\subsection{Mandel's problem}

Mandel's problem (see [45]) is an important benchmark problem because the analytical solution in two dimensions on a finite domain is known. It is an excellent model that can be used to verify the accuracy of a discretization. Mandel's problem models an infinitely long poroelastic slab sandwiched at the top and the bottom by two rigid frictionless and impermeable plates. The material is assumed incompressible and saturated with a single-phase 


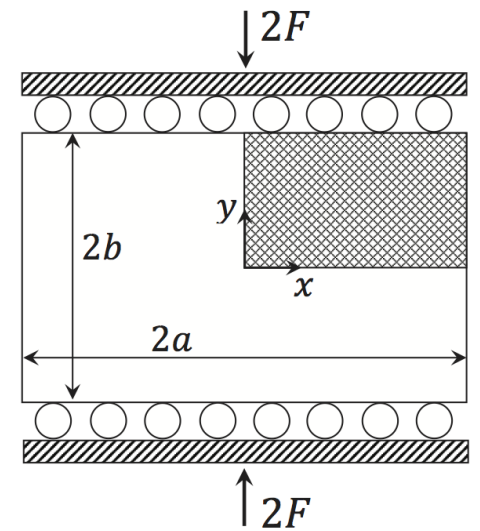

Figure 4.4: 2D physical and computational domains for Mandel's problem.

incompressible fluid. Both plates are loaded by a constant vertical force as shown in Figure 4.4, where a $2 a \times 2 b$ wide cross-section is displayed. The force of magnitude $2 F$ per unit length is suddenly applied at $t=0$, generating an instantaneous overpressure by the Skempton effect [46], which will dissipate near the side edges as time progresses due to the drainage effect, since the side surfaces $(x= \pm a)$ are drained and traction-free. In this problem, it turns out that the horizontal displacement $u$ is independent of the vertical direction $y$, whereas the vertical displacement $v$ is independent of the horizontal coordinate $x$. The analytical solution for the pore pressure can be found in [47] and is given as follows

$$
p(x, y, t)=2 * p_{0} \sum_{n=1}^{\infty} \frac{\sin \alpha_{n}}{\alpha_{n}-\sin \alpha_{n} \cos \alpha_{n}}\left(\cos \frac{\alpha_{n} x}{a}-\cos \alpha_{n}\right) \exp \left(\frac{-\alpha_{n}^{2} c t}{a^{2}}\right),
$$

where $p_{0}=\frac{1}{3 a} B\left(1+\nu_{u}\right) F$, being $B$ the Skempton's coefficient that for our problem is $B=1$ and $\nu_{u}=\frac{3 \nu+B(1-2 \nu)}{3-B(1-2 \nu)}$ the undrained Poisson's ratio, $c$ is the consolidation coefficient given by $c=K(\lambda+2 \mu)$, and $\alpha_{n}$ are the positive roots of the nonlinear equation

$$
\tan \alpha_{n}=\frac{1-\nu}{\nu_{u}-\nu} \alpha_{n}
$$

As can be observed in (43), also the pressure is independent of the vertical direction. In fact, Coussy (see [10]) shows that the normalized pressure is 
the solution of the following equation

$$
\frac{\partial \hat{p}}{\partial \hat{t}}-\frac{\partial^{2} \hat{p}}{\partial \hat{x}^{2}}=2 \sum_{n=1}^{\infty} \frac{\alpha_{n}^{2} \sin \alpha_{n} \cos \alpha_{n}}{\alpha_{n}-\sin \alpha_{n} \cos \alpha_{n}} \exp \left(-\alpha_{n}^{2} \hat{t}\right) .
$$

Note that the right-hand side is constant in space and it can become large at the beginning of the process.

For the finite element solution, the symmetry of the problem allows us to choose only a quarter of the physical domain as a computational domain, as shown in Figure 4.4. Moreover, the rigid plate condition is enforced by adding constrained equations such that vertical displacements on the top are equal to an unknown constant value. The triangulation of the computational domain is obtained from a uniform rectangular grid $n_{x} \times n_{y}$ by splitting each element in half. The dimension of the porous slab is specified by $a=b=1$, and the material properties are given by $K=10^{-6}, E=10^{4}, \nu=0$, and therefore $\nu_{u}=0.5$. The Lamè coefficients are computed in terms of the Young modulus and the Poisson ratio as follows,

$$
\lambda=\frac{E \nu}{(1-2 \nu)(1+\nu)}, \quad \mu=\frac{E}{2(1+\nu)} .
$$

Finally, the applied force has a magnitude of $F=1$.

The first test with Mandel's problem will illustrate the need of stabilizing the P1-P1 discretization, as well as the MINI element discretization, in order to remove the spurious oscillations in the pressure field. We choose a final time $T=10^{-4}$ for the computations with only one time-step, and a spatial grid with $n_{x}=n_{y}=32$. Since the pressure unknown is independent of the vertical coordinate, we will present the results on a representative horizontal line. In Figures 4.5 (a) and 4.5 (b), we show the numerical solution for the pressure (plotted in circular symbols) obtained by using P1-P1 finite element methods without and with stabilization, respectively. The numerical solution is plotted against the analytical solution that is displayed by a dashed line. The same comparison is shown in Figures 4.6 (a) and 4.6 (b) for the MINI element scheme. For the latter, the inf-sup condition is satisfied, but we observe that nonphysical oscillations appear in the pressure field, albeit smaller than in the P1-P1 case. By adding in both methods stabilization terms, oscillation-free solutions are obtained, as seen in Figures 4.5(b) and 4.6(b). Next, we analyze the behavior of the pressure in different times. For this purpose, in Figure 4.7 the solution of the pressure obtained by stabilized 


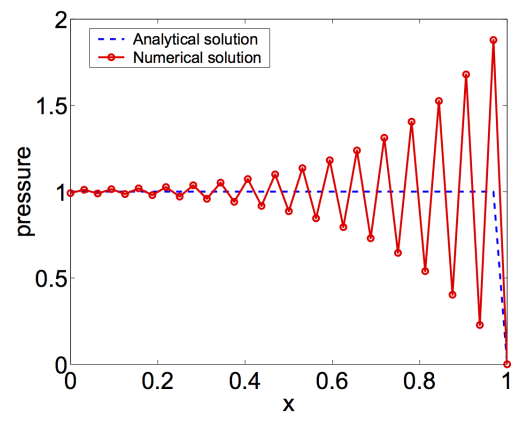

(a)

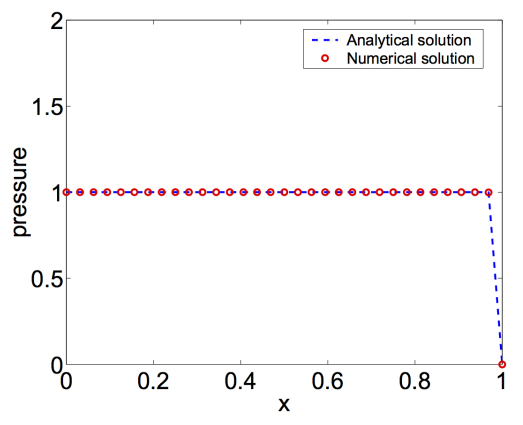

(b)

Figure 4.5: Numerical solution by P1-P1 of the pressure for Mandel's problem (a) without stabilization term and (b) with stabilization term.

P1-P1 finite elements on a grid with $n_{x}=n_{y}=32$, together with the corresponding analytical solution are shown in different times. We can observe a good agreement between both solutions for all the cases. A very interesting behavior of the solution of Mandel's problem is that it can achieve values greater than one at some time instants, which is known in the literature as the Mandel-Cryer effect. It is clear that this phenomenon is due to the source term that appears in equation (44), and is fully in agreement with the maximum principle for the heat equation.

To show the convergence properties of the stabilized schemes discussed earlier we compare the analytical solution, given in (43), with the numerical solution. For our tests, we choose progressively refined computational grids with mesh sizes $h_{k}=2^{-k} h_{0}, h_{0}=0.1$, and time steps $\tau_{k}=2^{-k} \tau_{0}$, with $\tau_{0}=0.5 T, k=0,1,2,3$, and where $T$ is the final time. In Figure 4.8 (a), for each mesh and a final time of $T=1$, we display the error for the pressure in the norm

$$
\left\|p\left(t_{n}\right)-p_{h}^{n}\right\|^{2}=\left\|p\left(t_{n}\right)-p_{h}^{n}\right\|^{2}+K \tau\left\|\nabla\left(p\left(t_{n}\right)-p_{h}^{n}\right)\right\|^{2} .
$$

In Figure 4.8 (a) we observe first order convergence, which confirms the error estimate obtained in Theorem 5. Next, we fix the size of the spatial grid in each direction to $h=1 / 80$, and we plot the pressure errors for different values of the time step, as shown in Figure 4.8 (b). Again, we observe a reduction of the error by a factor of two, and thus the corresponding first order convergence. Finally, we fix the value of the time discretization param- 


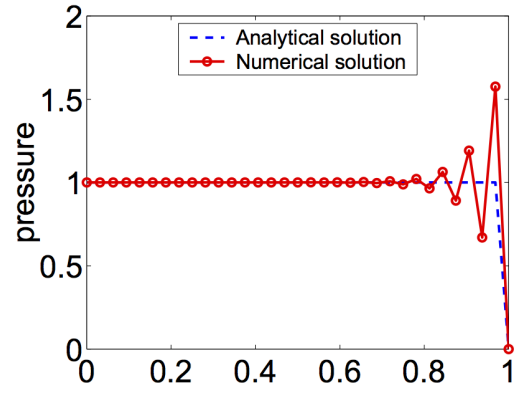

(a)

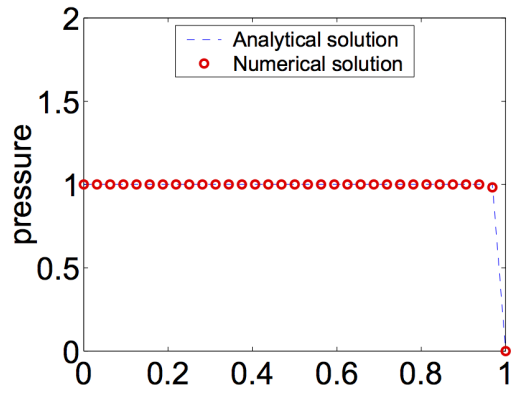

(b)

Figure 4.6: Numerical solution by MINI element of the pressure for Mandel's problem (a) without stabilization term and (b) with stabilization term.

eter to $\tau=2^{-10}$ and display in Figure 4.8 (c) the pressure errors for grids with different resolution $h_{k}=2^{-k} h_{0}, h_{0}=0.1$ and $k=0, \ldots, 3$. In such case, we observe a second order convergence in energy norm. This is a superconvergence and for finite difference discretizations, such results are found in [20]. This is a display of the well known superconvergence phenomena and is due to the fact that we have smooth exact solution and uniform grids. In the three figures we show only one convergence profile, because the results for both linear FEM and MINI element are essentially the same. This is due to the fact that very similar stabilization parameters have to be added to both methods to avoid the nonphysical oscillations, since the addition of the bubble plays a positive role but with a very small contribution. This point could be a reason to support the use of the stabilized P1-P1 scheme against the MINI element that also has to be stabilized.

\subsection{Barry \&3 Mercer's problem}

Another well-known benchmark problem on a finite two-dimensional domain is Barry \& Mercer's model, see [11]. It models the behavior of a rectangular uniform porous material with a pulsating point source, drained on all sides, and on which zero tangential displacements are assumed on the whole boundary. The point-source corresponds to a sine wave on the rectangular domain $[0, a] \times[0, b]$ and is given as follows

$$
f(t)=2 \beta \delta_{\left(x_{0}, y_{0}\right)} \sin (\beta t),
$$




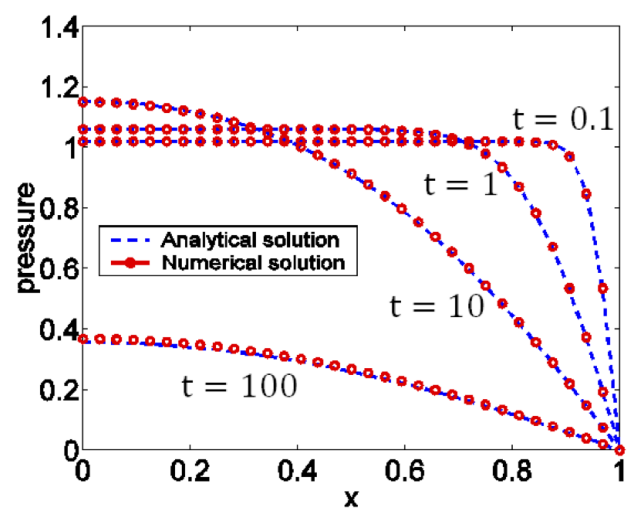

Figure 4.7: Comparison of numerical and analytical solutions of the pore pressure for Mandel's problem at various times.

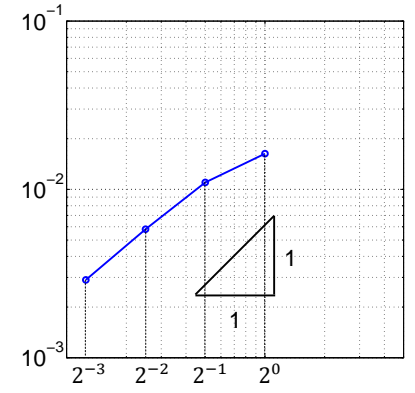

(a)

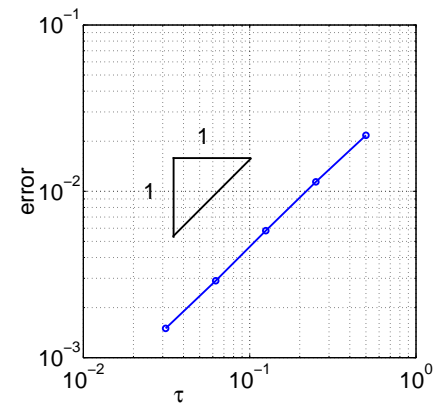

(b)

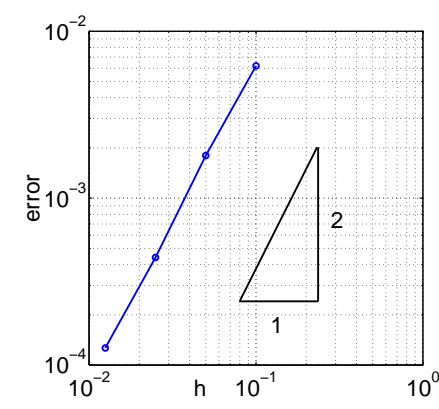

(c)

Figure 4.8: Pressure errors in energy norm (a) for progressively reduced values of the discretization parameters: $2^{-k} h_{0}$ and $2^{-k} \tau_{0}$ with $h_{0}=0.1, \tau_{0}=T / 2$ and $k=0,1,2,3$; (b) for a fixed value of $h=1 / 80$ and different values of $\tau=2^{-k} \tau_{0}$ with $k=0,1,2,3$; and (c) for a fixed value of $\tau=2^{-10}$ and different values of $h=2^{-k} / 10$ with $k=0,1,2,3$.

where $\beta=\frac{(\lambda+2 \mu) K}{a b}$ and $\delta_{\left(x_{0}, y_{0}\right)}$ is the Dirac delta at the point $\left(x_{0}, y_{0}\right)$. In Figure 4.9 the computational domain together with the boundary conditions are depicted. The boundary conditions do not correspond to a realistic physical situation, but they admit an analytical solution making this model a suitable test for numerical codes. Here we use this model to assess the monotone behavior of the approximations of the pressure.

We consider the rectangular domain $(0,1) \times(0,1)$, and the following values 


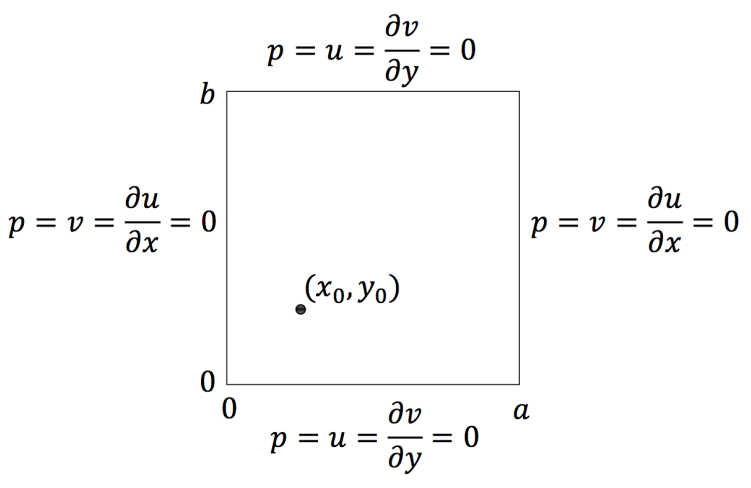

Figure 4.9: Computational domain and boundary conditions for the Barry and Mercer's source problem.

of the material parameters are considered $E=10^{5}, \nu=0.1$ and $K=10^{-2}$. The source is positioned at the point $(1 / 4,1 / 4)$ and a right triangular grid with $n_{x}=n_{y}=64$ is used for the simulations. The solution for the pressure produced by the stabilized P1-P1 scheme is plotted in Figure 4.10 for two different "normalized times" $\hat{t}=\beta t$ of values $\hat{t}=\pi / 2$ and $\hat{t}=3 \pi / 2$. Also we display the deformation of the considered triangular grid, according to the results obtained for the displacements. We can observe that depending on the sign of the source term (positive for $\hat{t}=\pi / 2$ and negative for $\hat{t}=3 \pi / 2$ ) the resultant displacements cause an expansion or a contraction of the medium.

The analytical solution of this problem is given by an infinite series, and can be found in [11]. It has been observed that solutions displayed in Figure 4.10 resemble the exact solution very precisely.

Fluid pressure oscillations for the Barry and Mercer's problem can be demonstrated by considering the standard schemes given by a P1-P1 or MINI element discretizations. In order to see this characteristic non-physical oscillatory behavior, a small permeability and/or a short time intervals are considered. Therefore, in the previous test, we have changed the value of $K$ to $10^{-6}$ and $T$ to $10^{-4}$. For these parameters, in Figure 4.11 we show the numerical solutions obtained for the pressure field, by using P1-P1 scheme (on the top) and the MINI element (on the bottom). We can observe that if no stabilization term is added to any of the discrete schemes (left pictures), then non-physical oscillations appear in the surroundings of the source-point. However, by adding the proposed artificial stabilizations, we can see (right 

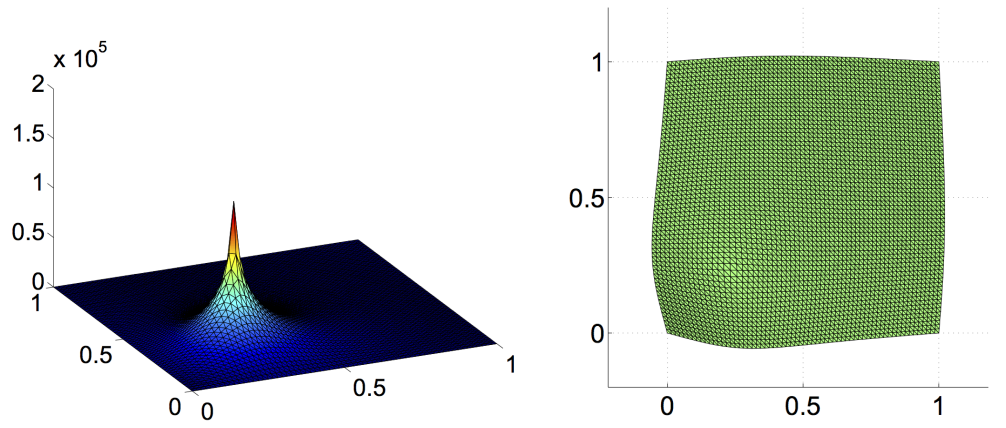

(a) $T=\pi / 2$
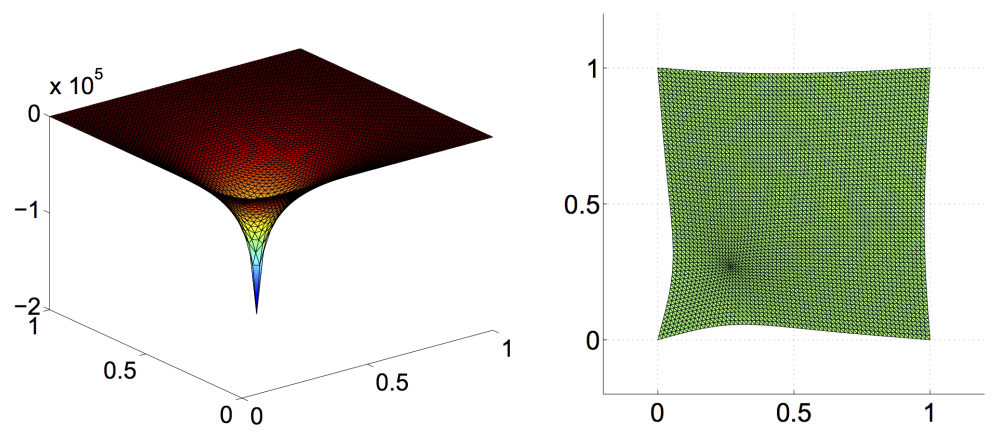

(b) $T=3 \pi / 2$

Figure 4.10: Numerical solution for the pressure by P1-P1 and deformation of the grid after applying the pulsating pressure point source, for two different values of $T$.

pictures) that these oscillations are completely eliminated.

\section{Conclusions}

In this paper we have analyzed the convergence and the monotonicity properties of low order discretizations of the Biot's consolidation model in poromechanics. While the convergence results are complete in some sense, there are still several open theoretical questions regarding the monotonicity of the resulting discretizations. Clearly, our numerical results show that choosing the stabilization parameters correctly lead to oscillation-free solutions, but justifying this rigorously is difficult and a topic of ongoing research. We have to say though that as a rule of thumb, one can choose stabilizations that are optimal in 1D, and, the resulting approximations in higher spatial 

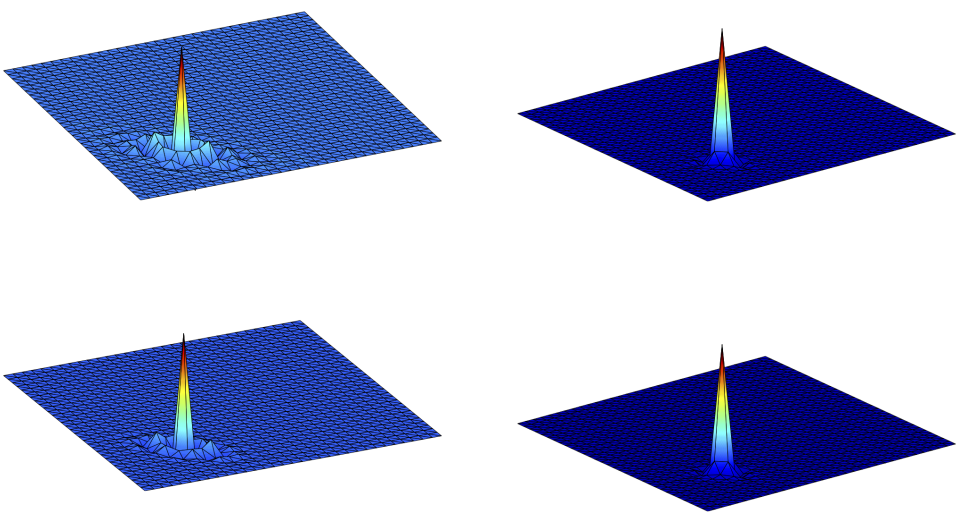

Figure 4.11: Numerical solution for the pressure field by P1-P1 (top) and MINI element (bottom), without and with stabilization term, at a final time of $10^{-4}$ and a permeability of $K=10^{-6}$.

dimensions will be oscillation-free.

\section{Acknowledgements}

The work of Francisco J. Gaspar and Carmen Rodrigo is supported in part by the Spanish project FEDER /MCYT MTM2013-40842-P and the DGA (Grupo consolidado PDIE). The research of Ludmil Zikatanov is supported in part by NSF DMS-1217142 and NSF DMS-1418843. Ludmil Zikatanov gratefully acknowledges the support for this work from the Institute of Mathematics and Applications at University of Zaragoza and Campus Iberus, Spain.

\section{Appendix A. Proof of Theorem 5}

In this appendix, we provide the proof of Theorem 5. First, we need the following lemma which estimates the error between the elliptic projection $\left\{\bar{u}_{h}\left(t_{n}\right), \bar{p}_{h}\left(t_{n}\right)\right\}$ and the numerical solutions $\left\{u_{h}^{n}, p_{h}^{n}\right\}$.

Lemma 7. Let $w_{u}^{j}:=\partial_{t} u\left(t_{j}\right)-\frac{\bar{u}_{h}\left(t_{j}\right)-\bar{u}_{h}\left(t_{j-1}\right)}{\tau}$ and $w_{p}^{j}:=\partial_{t} p\left(t_{j}\right)-\frac{\bar{p}_{h}\left(t_{j}\right)-\bar{p}_{h}\left(t_{j-1}\right)}{\tau}$, 
we have

$\left\|\left(e_{u}^{n}, e_{p}^{n}\right)\right\|_{\tau, h} \leq\left\|\left(e_{u}^{0}, e_{p}^{0}\right)\right\|_{\tau, h}+c \tau \sum_{j=1}^{n}\left(\left\|w_{u}^{j}\right\|_{a}+\varepsilon^{1 / 2} h\left\|\nabla w_{p}^{j}\right\|+\varepsilon^{1 / 2} h\left\|\nabla \partial_{t} p\left(t_{j}\right)\right\|\right)$.

If the initial data $u_{h}^{0}$ and $p_{h}^{0}$ satisfy (27) and (28), we have,

$$
\begin{aligned}
\left\|e_{p}^{n}\right\|_{a_{p}} \leq\left\|e_{p}^{0}\right\|_{a_{p}}+c \tau^{1 / 2}\left[\left(\sum_{j=1}^{n}\left\|w_{u}^{j}\right\|_{a}^{2}\right)^{1 / 2}\right. \\
\left.+\left(\sum_{j=1}^{n} \varepsilon h^{2}\left\|\nabla w_{p}^{j}\right\|^{2}\right)^{1 / 2}+\left(\sum_{j=1}^{n} \varepsilon h^{2}\left\|\nabla \partial_{t} p\left(t_{j}\right)\right\|^{2}\right)^{1 / 2}\right]
\end{aligned}
$$

and if the initial data $u_{h}^{0}$ and $p_{h}^{0}$ are defined by (29) and do not satisfy (27) and (28), we have,

$$
\begin{aligned}
\left\|e_{p}^{n}\right\|_{a_{p}} \leq \frac{1}{\sqrt{2 \tau}} & \left\|\left(e_{u}^{0}, e_{p}^{0}\right)\right\|_{\tau, h}+c \tau^{1 / 2}\left[\left(\sum_{j=1}^{n}\left\|w_{u}^{j}\right\|_{a}^{2}\right)^{1 / 2}\right. \\
& \left.+\left(\sum_{j=1}^{n} \varepsilon h^{2}\left\|\nabla w_{p}^{j}\right\|^{2}\right)^{1 / 2}+\left(\sum_{j=1}^{n} \varepsilon h^{2}\left\|\nabla \partial_{t} p\left(t_{j}\right)\right\|^{2}\right)^{1 / 2}\right]
\end{aligned}
$$

Moreover, we also have the following estimate in the $L^{2}$-norm,

$$
\left\|e_{p}^{n}\right\| \leq c\left\|\left(e_{u}^{0}, e_{p}^{0}\right)\right\|_{\tau, h}+c \tau \sum_{j=1}^{n}\left(\left\|w_{u}^{j}\right\|_{a}+\varepsilon^{1 / 2} h\left\|\nabla w_{p}^{j}\right\|+\varepsilon^{1 / 2} h\left\|\nabla \partial_{t} p\left(t_{j}\right)\right\|\right) .
$$

Proof. Choosing $v=v_{h} \in V_{h}$ in (20) and $q=q_{h} \in Q_{h}$ in (21), and subtracting both equations from (24) and (25), and we have for all $v_{h} \in V_{h}$ and $q_{h} \in Q_{h}$

$$
\begin{aligned}
& a\left(e_{u}^{n}, v_{h}\right)-\left(\operatorname{div} v_{h}, e_{p}^{n}\right)=0 \\
& \left(\operatorname{div} \bar{\partial}_{t} e_{u}^{n}, q_{h}\right)+a_{p}\left(e_{p}^{n}, q_{h}\right)+\varepsilon h^{2}\left(\nabla \bar{\partial}_{t} e_{p}^{n}, \nabla q_{h}\right) \\
& \quad=\left(\operatorname{div} w_{u}^{n}, q_{h}\right)+\varepsilon h^{2}\left(\nabla w_{p}^{n}, \nabla q_{h}\right)-\varepsilon h^{2}\left(\nabla \partial_{t} p\left(t_{n}\right), \nabla q_{h}\right)
\end{aligned}
$$


Choose $v_{h}=\bar{\partial}_{t} e_{u}^{n}$ in (A.5) and $q_{h}=e_{p}^{n}$ in (A.6) and add these two equations together, we have

$$
\begin{aligned}
\left\|\left(e_{u}^{n}, e_{p}^{n}\right)\right\|_{\tau, h}^{2} & =a\left(e_{u}^{n}, e_{u}^{n-1}\right)+\varepsilon h^{2}\left(\nabla e_{p}^{n}, \nabla e_{p}^{n-1}\right) \\
& +\tau\left(\operatorname{div} w_{u}^{n}, e_{p}^{n}\right)+\tau \varepsilon h^{2}\left(\nabla w_{p}^{n}, \nabla e_{p}^{n}\right) \\
& -\tau \varepsilon h^{2}\left(\nabla \partial_{t} p\left(t_{n}\right), \nabla e_{p}^{n}\right) \\
& \leq\left\|e_{u}^{n}\right\|_{a}\left\|e_{u}^{n-1}\right\|_{a}+\varepsilon h^{2}\left\|\nabla e_{p}^{n}\right\|\left\|\nabla e_{p}^{n-1}\right\| \\
& +\tau\left\|\operatorname{div} w_{u}^{n}\right\|\left\|e_{p}^{n}\right\|+\tau \varepsilon h^{2}\left\|\nabla w_{p}^{n}\right\|\left\|\nabla e_{p}^{n}\right\|+\tau \varepsilon h^{2}\left\|\nabla \partial_{t} p\left(t_{n}\right)\right\|\left\|\nabla e_{p}^{n}\right\|
\end{aligned}
$$

Thanks to the inf-sup condition (6), and (A.5) we have

$$
\begin{aligned}
\left\|e_{p}^{n}\right\| & \leq c \sup _{v_{h} \neq 0} \frac{\left(\operatorname{div} v_{h}, e_{p}^{n}\right)}{\left\|v_{h}\right\|_{a}}+c_{1} \varepsilon^{1 / 2} h\left\|\nabla e_{p}^{n}\right\| \\
& =c \sup _{v_{h} \in V_{h}} \frac{a\left(e_{u}^{n}, v_{h}\right)}{\left\|v_{h}\right\|_{a}}+c_{1} \varepsilon^{1 / 2} h\left\|\nabla e_{p}^{n}\right\|=c\left\|e_{u}^{n}\right\|_{a}+c_{1} \varepsilon^{1 / 2} h\left\|\nabla e_{p}^{n}\right\|(\mathrm{A} .8)
\end{aligned}
$$

Note, for MINI element, we have $c_{1}=0$ and, for P1-P1 element, $c_{1}>0$. Therefore,

$$
\begin{aligned}
\left\|\left(e_{u}^{n}, e_{p}^{n}\right)\right\|_{\tau, h}^{2} \leq \| & e_{u}^{n}\left\|_{a}\right\| e_{u}^{n-1}\left\|_{a}+\varepsilon h^{2}\right\| \nabla e_{p}^{n}\|\| \nabla e_{p}^{n-1} \| \\
& +c \tau\left\|w_{u}^{n}\right\|_{a}\left(\left\|e_{u}^{n}\right\|_{a}+c_{1} \varepsilon^{1 / 2} h\left\|\nabla e_{p}^{n}\right\|\right) \\
& +\tau \varepsilon h^{2}\left\|\nabla w_{p}^{n}\right\|\left\|\nabla e_{p}^{n}\right\|+\tau \varepsilon h^{2}\left\|\nabla \partial_{t} p\left(t_{n}\right)\right\|\left\|\nabla e_{p}^{n}\right\|
\end{aligned}
$$

which implies

$\left\|\left(e_{u}^{n}, e_{p}^{n}\right)\right\|_{\tau, h} \leq\left\|\left(e_{u}^{n-1}, e_{p}^{n-1}\right)\right\|_{\tau, h}+c \tau\left(\left\|w_{u}^{n}\right\|_{a}+\varepsilon^{1 / 2} h\left\|\nabla w_{p}^{n}\right\|+\varepsilon^{1 / 2} h\left\|\nabla \partial_{t} p\left(t_{n}\right)\right\|\right)$

We sum over all time steps and we have the estimate (A.1).

For the error estimate of $e_{p}^{n}$, from (A.5), we have,

$$
a\left(\bar{\partial}_{t} e_{u}^{n}, v_{h}\right)-\left(\operatorname{div} v_{h}, \bar{\partial}_{t} e_{p}^{n}\right)=0 .
$$

Note that, if the initial data $u_{h}^{0}$ and $p_{h}^{0}$ satisfy (27) and (28), (A.9) holds for $n=1,2,3, \ldots$ Otherwise, for initial data (29), (A.9) only holds for $n=2,3, \ldots$ 
Choosing $v_{h}=\bar{\partial}_{t} e_{u}^{n}$ in (A.9) and $q_{h}=\bar{\partial}_{t} e_{p}^{n}$ in (A.6) and adding the two equations, and we have

$$
\begin{aligned}
\tau^{-1} & \left\|e_{u}^{n}-e_{u}^{n-1}\right\|_{a}^{2}+\left\|e_{p}^{n}\right\|_{a_{p}}^{2}+\tau \varepsilon h^{2}\left\|\nabla \bar{\partial}_{t} e_{p}^{n}\right\|^{2} \\
\leq & \left\|e_{p}^{n}\right\|_{a_{p}}\left\|e_{p}^{n-1}\right\|_{a_{p}}+\left\|\operatorname{div} w_{u}^{n}\right\|\left\|e_{p}^{n}-e_{p}^{n-1}\right\| \\
& +\tau \varepsilon h^{2}\left\|\nabla w_{p}^{n}\right\|\left\|\nabla \bar{\partial}_{t} e_{p}^{n}\right\|+\tau \varepsilon h^{2}\left\|\nabla \partial_{t} p\left(t_{n}\right)\right\|\left\|\nabla \bar{\partial}_{t} e_{p}^{n}\right\| \\
\leq & \left\|e_{p}^{n}\right\|_{a_{p}}\left\|e_{p}^{n-1}\right\|_{a_{p}}+c\left\|\operatorname{div} w_{u}^{n}\right\|\left(\left\|e_{u}^{n}-e_{u}^{n-1}\right\|_{a}+c_{1} \varepsilon^{1 / 2} h\left\|\nabla\left(e_{p}^{n}-e_{p}^{n-1}\right)\right\|\right) \\
& +\tau \varepsilon h^{2}\left\|\nabla w_{p}^{n}\right\|\left\|\nabla \bar{\partial}_{t} e_{p}^{n}\right\|+\tau \varepsilon h^{2}\left\|\nabla \partial_{t} p\left(t_{n}\right)\right\|\left\|\nabla \bar{\partial}_{t} e_{p}^{n}\right\| \\
\leq & \frac{1}{2}\left\|e_{p}^{n}\right\|_{a_{p}}^{2}+\frac{1}{2}\left\|e_{p}^{n-1}\right\|_{a_{p}}^{2}+c \tau\left\|\operatorname{div} w_{u}^{n}\right\|^{2}+\tau^{-1}\left\|e_{u}^{n}-e_{u}^{n-1}\right\|_{a}^{2}+\frac{1}{3} \tau \varepsilon h^{2}\left\|\nabla \bar{\partial}_{t} e_{p}^{n}\right\|^{2} \\
& +\frac{3}{4} \tau \varepsilon h^{2}\left\|\nabla w_{p}^{n}\right\|^{2}+\frac{1}{3} \tau \varepsilon h^{2}\left\|\nabla \bar{\partial}_{t} e_{p}^{n}\right\|^{2}+\frac{3}{4} \tau \varepsilon h^{2}\left\|\nabla \partial_{t} p\left(t_{n}\right)\right\|^{2}+\frac{1}{3} \tau \varepsilon h^{2}\left\|\nabla \bar{\partial}_{t} e_{p}^{n}\right\|^{2},
\end{aligned}
$$

where we use the inf-sup condition (A.8) to estimate $\left\|e_{p}^{n}-e_{p}^{n-1}\right\|$. Now we have

$$
\left\|e_{p}^{n}\right\|_{a_{p}}^{2} \leq\left\|e_{p}^{n-1}\right\|_{a_{p}}^{2}+c\left(\tau\left\|w_{u}^{n}\right\|_{a}^{2}+\tau \varepsilon h^{2}\left\|\nabla w_{p}^{n}\right\|^{2}+\tau \varepsilon h^{2}\left\|\nabla \partial_{t} p\left(t_{n}\right)\right\|^{2}\right) .
$$

Now we need to consider two different cases due to the initial data. If the initial data satisfy (27) and (28), then above inequality (A.10) holds for $n=1$ and by summing up from 1 to $n$, we can get (A.2).

If the initial data is only defined by (29), (A.10) does not hold for $n=1$ anymore, we need to estimate $\left\|e_{p}^{1}\right\|$ separately. In order to do that, we take $n=1$ in (A.7) and then use the inf-sup condition (A.8) to estimate $\left\|e_{p}^{1}\right\|$,

$$
\begin{aligned}
\left\|e_{u}^{1}\right\|_{a}^{2}+\tau\left\|e_{p}^{1}\right\|_{a_{p}}^{2}+\varepsilon h^{2}\left\|\nabla e_{p}^{1}\right\|^{2} & =a\left(e_{u}^{1}, e_{u}^{0}\right)+\varepsilon h^{2}\left(\nabla e_{p}^{1}, \nabla e_{p}^{0}\right) \\
& +\tau\left(\operatorname{div} w_{u}^{1}, e_{p}^{1}\right)+\tau \varepsilon h^{2}\left(\nabla w_{p}^{1}, \nabla e_{p}^{1}\right) \\
& -\tau \varepsilon h^{2}\left(\nabla \partial_{t} p\left(t_{1}\right), \nabla e_{p}^{1}\right) \\
& \leq \frac{1}{2}\left\|e_{u}^{1}\right\|_{a}^{2}+\frac{1}{2}\left\|e_{u}^{0}\right\|_{a}^{2}+\frac{1}{2} \varepsilon h^{2}\left\|\nabla e_{p}^{1}\right\|^{2}+\frac{1}{2} \varepsilon h^{2}\left\|\nabla e_{p}^{0}\right\|^{2} \\
& +c \tau^{2}\left\|w_{u}^{1}\right\|_{a}^{2}+\frac{1}{2}\left\|e_{u}^{1}\right\|_{a}^{2}+\frac{1}{6} \varepsilon h^{2}\left\|\nabla e_{p}^{1}\right\|^{2}+\frac{3}{2} \tau^{2} \varepsilon h^{2}\left\|\nabla w_{p}^{1}\right\|^{2} \\
& +\frac{1}{6} \varepsilon h^{2}\left\|\nabla e_{p}^{1}\right\|^{2}+\frac{3}{2} \tau^{2} \varepsilon h^{2}\left\|\nabla \partial_{t} p\left(t_{1}\right)\right\|^{2}+\frac{1}{6} \varepsilon h^{2}\left\|\nabla e_{p}^{1}\right\|^{2} .
\end{aligned}
$$

This means

$$
\left\|e_{p}^{1}\right\|_{a_{p}}^{2} \leq \frac{1}{2 \tau}\left\|\left(e_{u}^{0}, e_{p}^{0}\right)\right\|_{\tau, h}+c\left(\tau\left\|w_{u}^{1}\right\|_{a}^{2}+\tau \varepsilon h^{2}\left\|\nabla w_{p}^{1}\right\|^{2}+\tau \varepsilon h^{2}\left\|\nabla \partial_{t} p\left(t_{1}\right)\right\|^{2}\right) .
$$


Now we summing up (A.10) from 2 to $n$ and use above estimate of $\left\|e_{p}^{1}\right\|_{a_{p}}^{2}$, we can get (A.3).

Finally, the estimate (A.4) follows directly from (A.1) and (A.8).

Next lemma give the estimations of $w_{u}^{j}$ and $w_{p}^{j}$.

Lemma 8. Let $u(t)$ and $p(t)$ be the solution of $(20)$ and $(21), w_{u}^{j}=\partial_{t} u\left(t_{j}\right)-$ $\frac{\bar{u}_{h}\left(t_{j}\right)-\bar{u}_{h}\left(t_{j-1}\right)}{\tau}$ and $\rho_{u}(t)=u(t)-\bar{u}_{h}(t)$. Assume $\partial_{t t} u(t) \in L^{1}\left((0, T],\left[H_{0}^{1}(\Omega)\right]^{d}\right) \cap$ $L^{2}\left((0, T],\left[H_{0}^{1}(\Omega)\right]^{d}\right)$ and $\partial_{t t} p(t) \in L^{1}\left((0, T], H_{0}^{1}(\Omega)\right) \cap L^{2}\left((0, T], H_{0}^{1}(\Omega)\right)$, we have,

$$
\begin{aligned}
& \sum_{j=1}^{n}\left\|w_{u}^{j}\right\|_{a} \leq c\left(\int_{0}^{t_{n}}\left\|\partial_{t t} u\right\|_{1} \mathrm{~d} t+\frac{1}{\tau} \int_{0}^{t_{n}}\left\|\partial_{t} \rho_{u}\right\|_{1} \mathrm{~d} t\right) \\
& \sum_{j=1}^{n}\left\|w_{u}^{j}\right\|_{a}^{2} \leq c\left(\tau \int_{0}^{t_{n}}\left\|\partial_{t t} u\right\|_{1}^{2} \mathrm{~d} t+\frac{1}{\tau} \int_{0}^{t_{n}}\left\|\partial_{t} \rho_{u}\right\|_{1}^{2} \mathrm{~d} t\right) .
\end{aligned}
$$

Moreover, let $w_{p}^{j}=\partial_{t} p\left(t_{j}\right)-\frac{\bar{p}_{h}\left(t_{j}\right)-\bar{p}_{h}\left(t_{j-1}\right)}{\tau}$ and $\rho_{p}=p(t)-\bar{p}_{h}(t)$. we have

$$
\begin{aligned}
& \sum_{j=1}^{n}\left\|\nabla w_{p}^{j}\right\| \leq c\left(\int_{0}^{t_{n}}\left\|\partial_{t} p\right\|_{1} \mathrm{~d} t+\frac{1}{\tau} \int_{0}^{t_{n}}\left\|\partial_{t} \rho_{p}\right\|_{1} \mathrm{~d} t\right) \\
& \sum_{j=1}^{n}\left\|\nabla w_{p}^{j}\right\|^{2} \leq c\left(\tau \int_{0}^{t_{n}}\left\|\partial_{t t} p\right\|_{1}^{2} \mathrm{~d} t+\frac{1}{\tau} \int_{0}^{t_{n}}\left\|\partial_{t} \rho_{p}\right\|_{1}^{2} \mathrm{~d} t\right) .
\end{aligned}
$$

Proof. We consider

$w_{u}^{j}=\left(\partial_{t} u\left(t_{j}\right)-\frac{u\left(t_{j}\right)-u\left(t_{j-1}\right)}{\tau}\right)+\left(\frac{u\left(t_{j}\right)-u\left(t_{j-1}\right)}{\tau}-\frac{\bar{u}_{h}\left(t_{j}\right)-\bar{u}_{h}\left(t_{j-1}\right)}{\tau}\right)=: w_{u, 1}^{j}+w_{u, 2}^{j}$.

Note that

$$
\begin{aligned}
w_{u, 1}^{j} & =\frac{1}{\tau} \int_{t_{j-1}}^{t_{j}}\left(s-t_{j-1}\right) \partial_{t t} u(s) \mathrm{d} s \\
w_{u, 2}^{j} & =\frac{1}{\tau} \int_{t_{j-1}}^{t_{j}} \partial_{t} \rho_{u}(s) \mathrm{d} s
\end{aligned}
$$


then we have

$$
\begin{aligned}
\left\|w_{u}^{j}\right\|_{a} & \leq\left\|w_{u, 1}^{j}\right\|_{a}+\left\|w_{u, 2}^{j}\right\|_{a} \\
& =\frac{1}{\tau}\left\|\int_{t_{j-1}}^{t_{j}}\left(s-t_{j-1}\right) \partial_{t t} u(s) \mathrm{d} s\right\|_{a}+\frac{1}{\tau}\left\|\int_{t_{j-1}}^{t_{j}} \partial_{t} \rho_{u}(s) \mathrm{d} s\right\|_{a} \\
& \leq c\left(\int_{t_{j-1}}^{t_{j}}\left\|\partial_{t t} u\right\|_{1} \mathrm{~d} s+\frac{1}{\tau} \int_{t_{j-1}}^{t_{j}}\left\|\partial_{t} \rho_{u}\right\|_{1} \mathrm{~d} s\right)
\end{aligned}
$$

then (A.11) follows directly. Moreover, we have

$$
\begin{aligned}
\left\|w_{u}^{j}\right\|_{a}^{2} & \leq c\left[\tau^{1 / 2}\left(\int_{t_{j-1}}^{t_{j}}\left\|\partial_{t t} u\right\|_{1}^{2} \mathrm{~d} s\right)^{1 / 2}+\tau^{-1 / 2}\left(\int_{t_{j-1}}^{t_{j}}\left\|\partial_{t} \rho_{u}\right\|_{1}^{2} \mathrm{~d} s\right)^{1 / 2}\right]^{2} \\
& \leq c\left(\tau \int_{t_{j-1}}^{t_{j}}\left\|\partial_{t t} u\right\|_{1}^{2} \mathrm{~d} s+\frac{1}{\tau} \int_{t_{j-1}}^{t_{j}}\left\|\partial_{t} \rho_{u}\right\|_{1}^{2} \mathrm{~d} s\right)
\end{aligned}
$$

then (A.12) follows directly. Estimates (A.13) and (A.14) can be obtained similarly, which completes the proof

Finally, we give the proof of Theorem 5 .

Proof. Proof of Theorem 5 The estimate (37) follows directly from (32), (33), (34), (35), (A.1), (A.11), (A.13), and triangle inequality. Note that we used (34) and (35) not only for $u, p$, but also their counterparts for $\partial_{t} \rho_{u}$ and $\partial_{t} \rho_{p}$.

Similarly, (38) follows from (33), (A.2), (A.12), (A.14), (34), (35), and their versions for the time derivatives of the error and the triangle inequality.

Next, for the second set of initial conditions, (39) follows from (33), (A.3), (A.12), (A.14), (34), (35) (applied also for time derivatives of the error), and the triangle inequality.

Finally, (40) follows from (33), (36), (A.4), (A.11), (A.13) and the triangle inequality.

\section{References}

[1] K. Terzaghi, Theoretical Soil Mechanics, Wiley: New York, 1943.

[2] M. A. Biot, General theory of threedimensional consolidation, Journal of Applied Physics 12 (2) (1941) 155-164. 
[3] M. A. Biot, Theory of elasticity and consolidation for a porous anisotropic solid, Journal of Applied Physics 26 (2) (1955) 182-185.

[4] R. Showalter, Diffusion in poro-elastic media, Journal of Mathematical Analysis and Applications 251 (1) (2000) 310 - 340.

[5] A. Ženíšek, The existence and uniqueness theorem in Biot's consolidation theory, Apl. Mat. 29 (3) (1984) 194-211.

[6] R. Z. Dautov, M. I. Drobotenko, A. D. Lyashko, Study on well-posedness of the generalized solution of the problem of filtration consolidation, Differents. Uravnenia 33 (1997) 515 - 521.

[7] T. Roose, P. A. Netti, L. L. Munn, Y. Boucher, R. K. Jain, Solid stress generated by spheroid growth estimated using a linear poroelasticity model, Microvascular Research 66 (3) (2003) $204-212$.

[8] C. Swan, R. Lakers, R. Brand, K. Stewart, Micromechanically based poroelastic modeling of fluid flow in haversian bone, J. Biomech. Eng. 125 (2003) $25-37$.

[9] A. D. Amit Halder, A. K. Datta, Modeling transport in porous media wiith phase change: Applications to food processing, J. Heat Transfer 133 (2010) 031010-1 - 031010-13.

[10] O. Coussy, Poromechanics, John Wiley \& Sons, Ltd, 2004.

[11] S. I. Barry, G. N. Mercer, Exact Solutions for Two-Dimensional TimeDependent Flow and Deformation Within a Poroelastic Medium, Journal of Applied Mechanics 66 (1999) 536. doi:10.1115/1.2791080.

[12] R. Lewis, B. Schrefler, The Finite Element Method in the Static and Dynamic Deformation and Consolidation of Porous Media, Wiley: New York, 1998.

[13] R. W. Lewis, B. Schrefler, A fully coupled consolidation model of the subsidence of venice, Water Resources Research 14 (2) (1978) 223-230.

[14] R. W. Lewis, D. V. Tran, Numerical simulation of secondary consolidation of soil: Finite element application, International Journal for $\mathrm{Nu}-$ merical and Analytical Methods in Geomechanics 13 (1) (1989) 1-18. 
[15] R. W. Lewis, B. A. Schrefler, L. Simoni, Coupling versus uncoupling in soil consolidation, International Journal for Numerical and Analytical Methods in Geomechanics 15 (8) (1991) 533-548.

[16] I. Masters, W. K. S. Pao, R. W. Lewis, Coupling temperature to a double-porosity model of deformable porous media, International Journal for Numerical Methods in Engineering 49 (3) (2000) 421-438.

[17] F. J. Gaspar, F. J. Lisbona, P. N. Vabishchevich, Finite difference schemes for poro-elastic problems, Comput. Methods Appl. Math. 2 (2) (2002) 132-142. doi:10.2478/cmam-2002-0008.

URL http://dx.doi.org/10.2478/cmam-2002-0008

[18] R. E. Ewing, O. P. Iliev, R. D. Lazarov, A. Naumovich, On convergence of certain finite volume difference discretizations for 1d poroelasticity interface problems, Numerical Methods for Partial Differential Equations 23 (3) (2007) 652-671.

[19] A. Naumovich, Efficient numerical methods for the Biot poroelasticity system in multilayered domains, Kaiserslautern, Techn. Univ., Diss., 2007.

[20] F. J. Gaspar, F. J. Lisbona, P. N. Vabishchevich, A finite difference analysis of Biot's consolidation model, Appl. Numer. Math. 44 (4) (2003) 487-506. doi:10.1016/S0168-9274(02)00190-3. URL http://dx.doi.org/10.1016/S0168-9274(02)00190-3

[21] M. Ferronato, N. Castelletto, G. Gambolati, A fully coupled 3-d mixed finite element model of Biot consolidation, J. Comput. Phys. 229 (12) (2010) 4813-4830.

[22] J. B. Haga, H. Osnes, H. P. Langtangen, On the causes of pressure oscillations in low-permeable and low-compressible porous media, International Journal for Numerical and Analytical Methods in Geomechanics 36 (12) (2012) 1507-1522. doi:10.1002/nag.1062.

[23] M. Favino, A. Grillo, R. Krause, A stability condition for the numerical simulation of poroelastic systems, in: C. Hellmich, B. Pichler, D. Adam (Eds.), Poromechanics V: Proceedings of the Fifth Biot Conference on Poromechanics, 2013, pp. 919-928.

URL http://ascelibrary.org/doi/abs/10.1061/9780784412992.110 
[24] P. Phillips, M. Wheeler, Overcoming the problem of locking in linear elasticity and poroelasticity: an heuristic approach, Computational Geosciences 13 (1) (2009) 5-12.

[25] F. Brezzi, On the existence, uniqueness and approximation of saddlepoint problems arising from Lagrangian multipliers, Rev. Française Automat. Informat. Recherche Opérationnelle Sér. Rouge 8 (R-2) (1974) $129-151$.

[26] M. A. Murad, A. F. D. Loula, Improved accuracy in finite element analysis of Biot's consolidation problem, Comput. Methods Appl. Mech. Engrg. 95 (3) (1992) 359-382. doi:10.1016/0045-7825(92)90193-N. URL http://dx.doi.org/10.1016/0045-7825(92)90193-N

[27] M. A. Murad, A. F. D. Loula, On stability and convergence of finite element approximations of Biot's consolidation problem, Internat. J. Numer. Methods Engrg. 37 (4) (1994) 645-667. doi:10.1002/nme.1620370407. URL http://dx.doi.org/10.1002/nme.1620370407

[28] M. A. Murad, V. Thomée, A. F. D. Loula, Asymptotic behavior of semidiscrete finite-element approximations of Biot's consolidation problem, SIAM J. Numer. Anal. 33 (3) (1996) 1065-1083. doi:10.1137/0733052.

URL http://dx.doi.org/10.1137/0733052

[29] G. Aguilar, F. Gaspar, F. Lisbona, C. Rodrigo, Numerical stabilization of Biot's consolidation model by a perturbation on the flow equation, Internat. J. Numer. Methods Engrg. 75 (11) (2008) 1282-1300. doi:10.1002/nme.2295.

URL http://dx.doi.org/10.1002/nme.2295

[30] C. Taylor, P. Hood, A numerical solution of the Navier-Stokes equations using the finite element technique, Internat. J. Comput. \& Fluids 1 (1) (1973) 73-100.

[31] P. A. Vermeer, A. Verruijt, An accuracy condition for consolidation by finite elements, International Journal for Numerical and Analytical Methods in Geomechanics 5 (1) (1981) 1-14. doi:10.1002/nag.1610050103. URL http://dx.doi.org/10.1002/nag.1610050103 
[32] D. N. Arnold, F. Brezzi, M. Fortin, A stable finite element for the Stokes equations, Calcolo 21 (4) (1984) 337-344 (1985). doi:10.1007/BF02576171.

URL http://dx.doi.org/10.1007/BF02576171

[33] F. Brezzi, M. Fortin, Mixed and hybrid finite element methods, Springer, New York, 1991.

[34] D. Boffi, F. Brezzi, M. Fortin, Mixed finite element methods and applications, Vol. 44 of Springer Series in Computational Mathematics, Springer, Heidelberg, 2013. doi:10.1007/978-3-642-36519-5.

URL http://dx.doi.org/10.1007/978-3-642-36519-5

[35] W. Zulehner, Nonstandard norms and robust estimates for saddle point problems, SIAM J. Matrix Anal. Appl. 32 (2) (2011) 536-560. doi:10.1137/100814767.

URL http://dx.doi.org/10.1137/100814767

[36] R. Stenberg, A technique for analysing finite element methods for viscous incompressible flow, Internat. J. Numer. Methods Fluids 11 (6) (1990) 935-948, the Seventh International Conference on Finite Elements in Flow Problems (Huntsville, AL, 1989). doi:10.1002/fld.1650110615.

URL http://dx.doi.org/10.1002/fld.1650110615

[37] F. Brezzi, J. Pitkäranta, On the stabilization of finite element approximations of the Stokes equations, in: Efficient solutions of elliptic systems (Kiel, 1984), Vol. 10 of Notes Numer. Fluid Mech., Friedr. Vieweg, Braunschweig, 1984, pp. 11-19.

[38] R. Pierre, Regularization procedures of mixed finite element approximations of the Stokes problem, Numer. Methods Partial Differential Equations 5 (3) (1989) 241-258. doi:10.1002/num.1690050307.

URL http://dx.doi.org/10.1002/num.1690050307

[39] R. Verfürth, Error estimates for a mixed finite element approximation of the Stokes equations, RAIRO Anal. Numér. 18 (2) (1984) 175-182.

[40] C. Rodrigo, F. Gaspar, X. Hu, L. T. Zikatanov, Stability and Monotonicity for Some Discretizations of the Biot's Model (Apr. 2015). arXiv:1504.07150. 
[41] R. E. Bank, B. D. Welfert, A comparison between the mini-element and the Petrov-Galerkin formulations for the generalized Stokes problem, Comput. Methods Appl. Mech. Engrg. 83 (1) (1990) 61-68. doi:10.1016/0045-7825(90)90124-5. URL http://dx.doi.org/10.1016/0045-7825(90)90124-5

[42] T. J. R. Hughes, L. P. Franca, M. Balestra, A new finite element formulation for computational fluid dynamics. V. Circumventing the Babuška-Brezzi condition: a stable Petrov-Galerkin formulation of the Stokes problem accommodating equal-order interpolations, Comput. Methods Appl. Mech. Engrg. 59 (1) (1986) 85-99. doi:10.1016/0045$7825(86) 90025-3$.

URL http://dx.doi.org/10.1016/0045-7825(86)90025-3

[43] F. Brezzi, J. Douglas, Jr., Stabilized mixed methods for the Stokes problem, Numer. Math. 53 (1-2) (1988) 225-235. doi:10.1007/BF01395886. URL http://dx.doi.org/10.1007/BF01395886

[44] V. Thomée, Galerkin finite element methods for parabolic problems, 2nd Edition, Vol. 25 of Springer Series in Computational Mathematics, Springer-Verlag, Berlin, 2006.

[45] J. Mandel, Consolidation des sols (étude de mathématique), Géotechnique 3 (1953) 287-299.

[46] A. W. Skempton, The pore-pressure coefficients A and B, Géotechnique 4 (1954) 143-147(4).

[47] Y. Abousleiman, A.-D. Cheng, L. Cui, E. Detournay, J.-C. Roegiers, Mandel's problem revisited, Geotechnique 46 (2) (1996) 187-195. 